\title{
Endemismo a diferentes escalas espaciales: un ejemplo con Carabidae (Coleoptera: Insecta) de América del Sur austral
}

\author{
Endemism at different spatial scales: an example with Carabidae (Coleoptera: Insecta) \\ of austral South America
}

CASAGRANDA M DOLORES ${ }^{1}$, SERGIO ROIG-JUNENT $^{2, *}$ \& CLAUDIA SZUMIK ${ }^{1}$

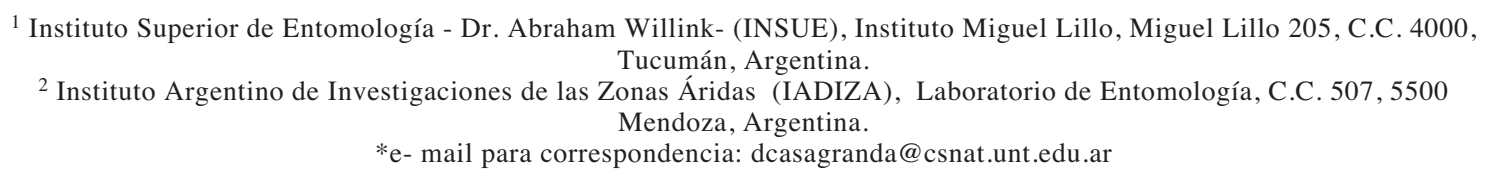

\section{RESUMEN}

Analizamos aproximadamente 4.000 registros correspondientes a 426 especies/subespecies de carábidos presentes en la región de América del Sur austral mediante análisis de endemicidad, considerando diferentes tamaños de celda. Para este análisis usamos el programa NDM/VNDM el cual implementa una función de llenado (R. fill) que permite inferir presencias potenciales de especies a partir de los datos de presencia observados. En este análisis exploramos el uso de diferentes valores de esta función y observamos sus efectos en los resultados obtenidos. Las áreas de endemismo encontradas se compararon con regionalizaciones biogeográficas propuestas previamente. Se obtuvieron numerosas áreas de endemismo, coincidentes en forma total o parcial con áreas naturales anteriormente descritas para la zona. Algunas de estas áreas fueron recuperadas en todos los tamaños de celda usados, mientras que otras solo se identificaron bajo un tamaño de celda específico. En general, el empleo de celdas pequeñas facilitó la identificación de áreas de endemismo disyuntas y áreas de corta extensión, mientras el uso de celdas mayores permitió la identificación de áreas de gran extensión geográfica que resultan fragmentadas al usar celdas más pequeñas. Con el aumento en los valores de R. fill usados se observó un incremento en el número de áreas de endemismo y especies endémicas. Nuestros resultados muestran que áreas de endemismo de diversas características se manifiestan al emplear diferentes combinaciones de R. fill y tamaño de grilla, enfatizando la importancia de explorar distintas opciones en los análisis durante la búsqueda de patrones de distribución.

Palabras clave: análisis de endemicidad, escala espacial, Carabidae, América del Sur austral.

\begin{abstract}
In this paper we perform an endemicity analysis using ca 4,000 distributional records from 426 carabid species/subspecies distributed along austral South America. We used the program NDM/VNDM which implements a fill function ( $R$. fill) to heuristically assign species' potential occurrence from observed presences. In the present analysis we use different grid sizes, and also we explore different values for the fill function ( $R$. fill). The areas of endemism identified by NDM/VNDM were compared with previous biogeographical hypotheses. Some resulting areas of endemism were recognized through the whole range of grid sizes, while others could only be identified by using a particular grid size. In general, the use of small cells helped identify disjunct areas of endemism, as well as small ones, whereas big cells were convenient for the identification of broad areas that appeared as fragmented if smaller cells were used. In general, as R.fill function increased, the number of recognized areas of endemism and endemic species also increased. Our results show that areas of endemism with diverse traits can be derived from a singular combination of R. fill and grid size, emphasizing the importance of exploring different analytical options during the identification of distributional patterns.
\end{abstract}

Key words: endemicity analysis, spatial scale, Carabidae, austral South America. 


\section{INTRODUCCIÓN}

El área que se extiende por debajo del paralelo $30^{\circ}$ de latitud sur, denominada América del Sur austral (Jeannel 1942, 1967, Crisci et al. 1991, Roig-Juñent 1994) se distingue notablemente del resto de la región neotropical por poseer una biota que la relaciona con las regiones circumantárticas. Esta singularidad de América del Sur austral ha sido documentada y discutida por especialistas notables desde el siglo XIX hasta el presente (e.g., Hooker 1853, Monrós 1958, Skottsberg 1960, Kuschel 1964, 1969, Darlington 1965, Brundin 1966; Humphries 1981). Aunque muchos de los estudios realizados en la región son descripciones de tipo cualitativas, en las últimas décadas se realizaron numerosos estudios que introducen el uso de metodologías cuantitativas. Si bien la mayoría de ellos analizan pequeñas porciones de esta gran región, escasean estudios que abarquen la región de América del Sur austral en forma completa.

Uno de los primeros autores en realizar estudios biogeográficos cuantitativos en esta región fue Artigas (1975) quien dividió Chile continental en celdas de igual tamaño y buscó relaciones entre las celdas mediante la aplicación de índices de similitud. En los últimos años se ha buscado identificar unidades biogeográficas en América del Sur austral aplicando análisis panbiogeográficos (Morrone et al. 2001a, 2001b, 2006), análisis de parsimonia de endemismos (Posadas \& Miranda-Esquivel 1999, Pizarro et al. 2004) y análisis de endemicidad (Szumik \& Roig 2005, Domínguez et al. 2006). Con excepción de los análisis panbiogeográficos, los restantes métodos utilizan como unidad de análisis cuadrículas de una grilla (celdas) que contienen información de presencia/ausencia de las especies analizadas. Las grillas dividen la región estudiada en áreas de superficie comparable y su uso como unidad básica de análisis es una manera simple de uniformar, cuantificar, y transformar la búsqueda de patrones en un proceso objetivo y repetible. $\mathrm{La}$ grilla utilizada más frecuentemente es la basada en un cuadriculado de meridianos y paralelos geográficos. Aunque las celdas de este tipo de grilla no poseen igual superficie, debido a la distorsión latitudinal, uno de los motivos por los cuales se usan comúnmente es la facilidad con que se construyen sobre datos de coordenadas espaciales (georreferencias).

A pesar de ser unidades operacionales objetivas, un punto muy debatido en torno al uso de celdas es que, a partir de los mismos datos de origen, pueden encontrarse diferentes patrones según el tamaño de celda empleado. Si bien este tema ha sido discutido por varios autores, especialmente en trabajos de índole ecológica (Anderson \& Marcus 1993, Laffan \& Crisp 2003), en biogeografía histórica la exploración del efecto producido por la variación de escala ha sido generalmente dejada de lado.

En el presente trabajo se realiza un análisis de endemicidad (Szumik et al. 2002, Szumik \& Goloboff 2004) sobre la base de datos de distribución de 426 especies/subespecies de carábidos (Coleoptera: Carabidae), utilizando diferentes escalas de análisis (tamaños de celdas) con el fin de identificar áreas de endemismo en América del Sur austral, y de observar los efectos producidos por la variación del tamaño de celda empleado en la identificación de estos patrones. Los resultados obtenidos se comparan con biorregiones previamente descritas para la zona de estudio y se discuten los efectos producidos por la variación de la escala de análisis así como de otros parámetros de búsqueda.

\section{MATERIALES Y MÉTODOS}

\section{Área de estudio}

América del Sur austral (Kuschel 1969, Jeannel 1967, Crisci et al. 1991) ocupa gran parte de la porción austral de Chile y Argentina. Esta región se extiende al sur del paralelo $31^{\circ} \mathrm{S}$ en Chile, y en Argentina ocupa el centro oeste del país, entre los paralelos $35-40^{\circ} \mathrm{S}$ y parte de la Patagonia al sur del paralelo $40^{\circ} \mathrm{S}$. Se incluyen también como parte de esta región varios sistemas montañosos extraandinos de Argentina (Roig-Juñent et al. 2003), y numerosas islas y archipiélagos de los océanos Pacífico y Atlántico (Juan Fernández, San Ambrosio, Chiloé, Chonos y Cabo de Hornos en el océano Pacífico, Isla de los Estados, Malvinas y Georgias del sur, en el Atlántico, y Tierra del Fuego con costas en ambos océanos). El área abarcada cubre la región andina, incluyendo las 
subregiones chilena central, subantártica, patagónica, y parte de la zona de transición sudamericana (sensu Morrone 2006).

\section{Datos}

El análisis incluye 3.989 registros georreferenciados correspondientes a 426 especies y subespecies de carábidos, pertenecientes a 82 géneros. A cada una de las especies se le asignó un número de identificación mediante el cual se hace referencia en el texto (Anexo 1). Los datos provienen de revisiones bibliográficas, y de las siguientes colecciones entomológicas: Chile: Museo Nacional de Historia Natural de Chile (MNHNC, Santiago), Museo de la Universidad Metropolitana de Ciencias de la Educación (UMCE, Santiago), Instituto de la Patagonia (Punta Arenas); Argentina: Museo de La Plata (MLP, La Plata), Museo Argentino de Ciencias Naturales Bernardino Rivadavia (MACN, Buenos Aires), Instituto Miguel Lillo (IML, Tucumán), Instituto Patagónico de Ciencias Naturales (IPCN, San Martín de los Andes), Instituto Argentino de Investigaciones de Zonas Áridas (IADIZA); Inglaterra: Natural History Museum, (NHM, Londres); Estados Unidos de América: American Museum of Natural History (AMNH, New York), National Museum of Natural History (NMNH, Smithsonian Institution, Washington).

\section{Variables}

La búsqueda de áreas de endemismo fue realizada siguiendo el análisis de endemicidad (AE) propuesto por Szumik et al. (2002) y Szumik \& Goloboff (2004), implementado en los programas NDM/VNDM (Goloboff 2004).

El procedimiento desarrollado en NDM/ VNDM para la identificación de áreas de endemismo consiste, básicamente, en evaluar la congruencia del rango de distribución de las especies a un área predefinida (conjunto de celdas). El ajuste de las especies al área es medido cuantitativamente a través de un índice de endemicidad $\left(\mathrm{IE}_{\mathrm{e}}\right)$ cuyos valores varían entre 0 y 1 ; donde $\mathrm{IE}_{\mathrm{e}}=1$ representa una especie cuyo rango de distribución es perfectamente congruente con el área evaluada ("endemismo perfecto"). Durante el cálculo del $\mathrm{IE}_{\mathrm{e}} \mathrm{se}$ consideran registros de ocurrencia observados (obtenidos a partir de información empírica concreta, e.g., datos provenientes de colecciones museológicas, bibliografía, etc.) así como registros potenciales, calculados según funciones de "llenado" implementadas en NDM/VNDM -o designados manualmente por el usuario-. Los registros potenciales pueden clasificarse según el modo en que son modelados en: (a) registros inferidos, calculados de manera automática por NDM/ VNDM según un criterio de proximidad y homogeneidad implementado en el programa. Según este criterio se atribuirá presencia potencial de un taxón en una celda dada, si dicha celda cuenta con un número mínimo de presencias observadas en su vecindad (Szumik \& Goloboff 2004). Los parámetros involucrados en esta función (i.e., número mínimo de celdas aledañas con presencia real) pueden ser modificados por el usuario. (b) Registros supuestos, donde las presencias supuestas se calculan mediante un radio de llenado (R. ass.) que determina la superficie alrededor de un punto de presencia observada de la especie, en que esta se asumirá presente (Szumik \& Goloboff 2004). Los valores de R. ass. pueden ser modificados por el usuario. Los registros potenciales modifican al $\mathrm{IE}_{\mathrm{e}}$ en menor medida que los registros reales. La magnitud con que estos registros intervienen en el $\mathrm{IE}_{\mathrm{e}}$ puede modificarse mediante factores de pesado (i.e., $F_{i}, F_{a}, F_{o}, F_{d}, F_{n}$ ). Los rangos de distribución de las especies pueden ser ampliados mediante una función de llenado que opera en forma análoga al modelado de presencias asumidas. Los registros obtenidos de esta forma se computarán como registros observados, aportando al $\mathrm{IE}_{\mathrm{e}}$ igual valor que estos; este tipo de "llenado" también es regulado mediante un valor de radio ( $\mathrm{R}$. fill). El $\mathrm{IE}_{\mathrm{e}}$ mide el ajuste de la distribución de una especie al área evaluada considerando los distintos tipos de registros mencionados, el según la siguiente fórmula:

$I E e=\frac{p+\left(i \times F_{i}\right)+\left(a \times F_{a}\right)}{T+\left(o \times 1 / F_{o}\right)+\left(d \times 1 / F_{d}\right)+\left(n \times 1 / F_{n}\right)}(0,1)$,

donde, $\mathrm{p}=$ número de celdas del área donde el taxón está presente, $\mathrm{i}=$ número de celdas del área donde el taxón está inferido, a = número de celdas del área donde el taxón está supuesto, $\mathrm{T}=$ número total de celdas que tiene el área, $\mathrm{o}=$ número de celdas adyacentes al área donde 
el taxón está presente, $\mathrm{d}=$ número de celdas adyacentes al área donde el taxón está supuesto, $\mathrm{n}=$ número de celdas alejadas del área donde el taxón $\mathrm{X}$ está supuesto, $\mathrm{F}_{\mathrm{i}}=$ factor de pesado para registros inferidos dentro del área (default $=0,50), \mathrm{F}_{\mathrm{a}}=$ factor para registros inferidos dentro del área (default $=0,75), \mathrm{F}_{\mathrm{o}}=$ factor para registros observados adyacentes al área (default $=0,50), \mathrm{F}_{\mathrm{d}}=$ factor para registros asumidos adyacentes al área (default $=2,00$ ), y $\mathrm{F}_{\mathrm{n}}=$ factor para registros asumidos alejados del área (default $=0,50)$.

En términos generales, registros de una especie distribuidos homogéneamente dentro del área evaluada aumentarán el $\mathrm{IE}_{\mathrm{e}}$ de tal especie, mientras que la presencia de registros fuera del área disminuirá su $\mathrm{IE}_{\mathrm{e}}$. El índice de endemicidad de un área (IEA) es igual a la suma de los $\mathrm{IE}_{\mathrm{e}}$ de sus especies endémicas, de manera que cuanto más especies endémicas presente un área, y cuanto mayor sea su grado de endemicidad, el grupo de celdas estará mejor apoyado como "área de endemismo" (Szumik \& Goloboff 2004).

Para la descripción y comparación de los resultados se utilizaron áreas de consenso (AC, Szumik \& Goloboff datos no publicados). Análogamente a los consensos de árboles en filogenia, las AC resumen información común contenida en las hipótesis individuales resultantes. Un AC reúne áreas de endemismo individuales (AI) que presentan un porcentaje (fijado por el usuario) de especies endémicas en común (i.e., AI similares en su composición de especies serán fusionadas en un AC). La similitud entre las diferentes AI puede ser medida de manera más o menos estricta, dando lugar a consensos "estrictos" o "flexibles" (ver Manual NDM/VNDM). La utilización de AC facilita la revisión y comparación de los resultados, mostrando en forma sintética los patrones generales encontrados.

En este trabajo utilizamos consensos flexibles, agrupando AI que compartan como mínimo un $40 \%$ de sus especies endémicas. Los resultados se organizaron siguiendo las clasificaciones biogeográficas propuestas por Morrone (2001a, 2001b, 2006), aunque los resultados también fueron comparados con propuestas de otros autores (Cabrera \& Willik 1973, Roig-Juñent 1994, Martínez-Carretero 1995, Roig-Juñent \& Flores 2001, Morrone et al. 2002, Domínguez et al. 2006).

\section{Análisis}

Los datos fueron analizados tomando como unidad diferentes rangos taxonómicos, a fin de explorar su efecto en el reconocimiento de patrones biogeográficos. Realizamos dos tipos de análisis: (i) análisis de endemicidad de especies: los datos de distribución correspondientes a diferentes especies fueron considerados en forma independiente -rangos de distribución de especies individuales-; (ii) análisis de endemicidad de géneros: los registros correspondientes a especies pertenecientes a un mismo género fueron agrupados, tratándose cada conjunto como una unidad (rangos de distribución de géneros).

Los casos mencionados fueron analizados bajo distintos tamaños de celda: $1^{\circ} \mathrm{X} 1^{\circ}$ (lat/ long), $0,5^{\circ} \times 0,5^{\circ}$ (lat/long), y $0,25^{\circ} \times 0,25^{\circ}$ (lat/long), con el fin de describir el efecto causado por esta variable (escala espacial) en la identificación de áreas de endemismo. Las correspondientes matrices se encuentran disponibles en el Apéndice 1.

Los parámetros de búsqueda (i.e., número mínimo de especies endémicas, valor mínimo de IE) se mantuvieron constantes en todos los análisis, mientras que los valores usados para el modelado de presencias potenciales (R.fill, R. ass.) fueron modificados según el tamaño de grilla. Los valores utilizados se muestran en la Tabla 1.

\section{RESULTADOS}

Las AC van seguidas de un subíndice que indica el tamaño de celda de la matriz de análisis, así $\mathrm{AC}_{0,25}$ representa un área de consenso proveniente del análisis de $0,25^{\circ} \mathrm{x}$ $0,25^{\circ}$, mientras $\mathrm{AC}_{0,5}$ y $\mathrm{AC}_{1}$ provienen de matrices de $0,5^{\circ}$ x $0,5^{\circ}$ y $1^{\circ}$ x $1^{\circ}$, respectivamente.

\section{Áreas de endemismo}

En total se obtuvieron $37 \mathrm{AC}$ : 10 AC obtenidas del análisis de $0,25^{\circ}$ x $0,25^{\circ}$ (Tabla 2) construidas a partir de $15 \mathrm{AI} ; 17 \mathrm{AC}$ del análisis de $0,5^{\circ} \times 0,5^{\circ}$ (Tabla 3 ) construidas a partir de $35 \mathrm{AI}$; y $20 \mathrm{AC}$ de $1^{\circ}$ x $1^{\circ}$ elaboradas en base a 116 AI (Tabla 4). 


\section{Subregión de Chile central}

En los análisis de menor escala se identifican tres AC dentro de la subregión de Chile central. El $A_{0,25} 9$ representa la provincia de Santiago en forma completa, e incluye como subáreas a $\mathrm{AC}_{0,25} 8$ y $\mathrm{AC}_{0,25} 2$ que se corresponden con la cordillera de la Costa, cordillera de los Andes y valle central (Roig-Juñent et al. 2005), respectivamente (Fig. 1A).
De manera similar a lo ocurrido en los análisis de $0,25^{\circ}$, en el análisis de $0,5^{\circ}$ se identifica la provincia de Santiago en forma completa $\left(\mathrm{AC}_{0,5} 8\right.$, Fig. 1B), incluyendo áreas de endemismo definidas por especies de distribución más restringida: $\mathrm{AC}_{0,5} 7$ y $\mathrm{AC}_{0,5}$ 14, que representan la cordillera de la Costa, la cordillera de los Andes y valle central, respectivamente. En esta escala se identifica una nueva área de endemismo coincidente con

TABLA 1

Parámetros (M, m, -p, \%) y radios de llenado (R. fill, R. ass.) usados durante los análisis de endemicidad a diferentes escalas espaciales y diferentes niveles taxonómicos: $\mathrm{M}=$ mínimo valor de endemicidad requerido para guardar un área como endémica; $m$ = número mínimo de especies endémicas requerido para guardar un área como endémica; - $p$ = usar "edge proportions"; \% = porcentaje de especies endémicas exclusivas requerido para guardar un área como endémica.

Parameters (M, m, -p, \%) and filling radius (R. fill, R. ass.) employed for endemicity analysis at different spatial scales and different taxonomic levels: $M=$ minimum value of endemicity required to save an area as endemic; $\mathrm{m}=$ minimum number of endemic species required to save an area as endemic; - $p=$ use "edge proportions"; $\%=$ percentage of exclusive endemic species required to save an area as endemic.

\begin{tabular}{|c|c|c|c|c|c|c|c|c|}
\hline \multirow[t]{2}{*}{ M } & \multirow[t]{2}{*}{$\mathrm{m}$} & \multirow[t]{2}{*}{$-p$} & \multirow[t]{2}{*}{$\%$} & & \multicolumn{2}{|c|}{ R. fill } & \multicolumn{2}{|c|}{ R. ass } \\
\hline & & & & & $\mathrm{x}$ & $\mathrm{y}$ & $\mathrm{x}$ & $\mathrm{Y}$ \\
\hline \multicolumn{9}{|c|}{ Análisis a nivel de especies } \\
\hline $0,25^{\circ} \times 0,25^{\circ}$ & 2 & 2 & $\mathrm{X}$ & 10 & 50 & 50 & 100 & 100 \\
\hline $0,50^{\circ} \times 0,50^{\circ}$ & 2 & 2 & $\mathrm{X}$ & 10 & 20 & 20 & 50 & 50 \\
\hline $1^{\circ} \times 1^{\circ}$ & 2 & 2 & $\mathrm{X}$ & 10 & 15 & 15 & 30 & 30 \\
\hline \multicolumn{9}{|c|}{ Análisis a nivel de género } \\
\hline $0,25^{\circ} \times 0,25^{\circ}$ & 2 & 2 & $\mathrm{X}$ & 10 & 50 & 50 & 100 & 100 \\
\hline $0,50^{\circ} \times 0,50^{\circ}$ & 2 & 2 & $\mathrm{X}$ & 10 & 25 & 25 & 60 & 60 \\
\hline $1^{\circ} \times 1^{\circ}$ & 2 & 2 & $\mathrm{X}$ & 10 & 15 & 15 & 30 & 30 \\
\hline
\end{tabular}

TABLA 2

Áreas de consenso (AC) obtenidas usando grillas de $0.25^{\circ} \times 0.25^{\circ}$. Para cada AC se indica: áreas individuales (AI) incluidas, especies endémicas presentes, tamaño del área (expresado en número de celdas).

Consensus areas (AC) obtained using grids of $0.25^{\circ} \times 0.25^{\circ}$. Individual areas (AI), endemic species, area size (expressed as cell numbers).

\begin{tabular}{lccc}
\hline Número de áreas de consenso & AI incluidas & Especies presentes & Tamaño del área \\
\hline 1 & $1-8-5$ & $92-98-100-158-238-314$ & 14 \\
2 & 2 & $139-155-255-355$ & 9 \\
3 & 3 & $185-187-188-189-190-233-302-305-306-328-362-364$ & 9 \\
4 & 4 & $186-304-361$ & 21 \\
5 & 6 & $193-259-290-291-389$ & 17 \\
6 & 7 & $122-129-197-309$ & 8 \\
7 & 9 & $191-192-336-352$ & 26 \\
8 & 10 & $70-140-178-234-379$ & 10 \\
9 & $11-12-13-14$ & $93-140-180-218-234-235-379-382-385-404$ & 20 \\
10 & 15 & $94-230-293-392$ & 8 \\
\hline
\end{tabular}


la provincia biogeográfica de Coquimbo $\left(\mathrm{AC}_{0,5}\right.$ 1, Tabla 3).

Los análisis de $1^{\circ} \times 1^{\circ}$ produjeron cinco $\mathrm{AC}$ $-\mathrm{AC}_{1} 1, \mathrm{AC}_{1}$ 6, $\mathrm{AC}_{1} 8, \mathrm{AC}_{1}$ 15, $\mathrm{AC}_{1}$ 17-(Fig. 1C) congruentes con la subregión chilena central. Con excepción de $\mathrm{AC}_{1} 17$, caracterizada por un conjunto de especies endémicas diferente, las restantes $\mathrm{AC}$ presentan entre sí un alto grado de superposición espacial y comparten numerosas especies (Tabla 4). En esta escala de análisis se identifican además dos $\mathrm{AC}$ de gran extensión geográfica $-\mathrm{AC}_{1} 3$ y $\mathrm{AC}_{1} 20$ (Fig. 1D)- que ocupan fracciones de diversas unidades biogeográficas (subregión Puneña (Prepuna), subregión chilena central, región subantártica (Maule y bosque valdiviano) y subregión patagónica, sensu Morrone (2001a)). La gran amplitud y heterogeneidad de estas áreas podrían reflejar cierto efecto del tamaño de grilla empleado (ver discusión).

\section{Subregión Subantártica}

En los análisis de $0,25^{\circ}$ se pudieron determinar áreas de endemismo coincidentes con tres provincias de la subregión Subantártica (Fig. 2). El $\mathrm{AC}_{0,25} 6$ (Fig. 2A) ocupa un pequeño sector de la selva valdiviana o provincia de bosque valdiviano (Morrone 2001a), el AC $_{0,25}$ 10 ocupa una porción de la Araucanía o provincia biogeográfica de Maule (Morrone 2001a, ver Fig. 2A), y $\mathrm{AC}_{0,25} 3, \mathrm{AC}_{0,25} 4$ y $\mathrm{AC}_{0,25} 7$ se corresponden con la provincia biogeográfica de Juan Fernández (Fig. 3A). Mientras $\mathrm{AC}_{0,25} 4$ (caracterizada por tres especies endémicas) identifica el Archipiélago de Juan Fernández en forma completa; $\mathrm{AC}_{0,25} 3$ (con 12 especies endémicas) y $\mathrm{AC}_{0,25} 7$ (4 especies endémicas) recuperan las islas Robinson Crusoe y Alejandro Selkirk en forma individual (Fig. 3A, Tabla 2).

TABLA 3

Áreas de consenso (AC) obtenidas usando grillas de $0,5^{\circ} \times 0,5^{\circ}$. Para cada $\mathrm{AC}$ se indica: áreas individuales (AI) incluidas especies endémicas presentes, tamaño del área (expresado en número de celdas).

Consensus areas (AC) obtained using grids of $0.5^{\circ} \times 0.5^{\circ}$. Included individual areas (AI), endemic species area size (expressed as cell numbers).

\begin{tabular}{|c|c|c|c|}
\hline $\begin{array}{l}\text { Número de áreas } \\
\text { de consenso }\end{array}$ & $\begin{array}{l}\text { Áreas individuales } \\
\text { incluidas }\end{array}$ & Especies presentes & Tamaño del área \\
\hline 1 & 1 & $68-84-130$ & 4 \\
\hline 2 & $2-18$ & $92-98-100-157-160-238$ & 7 \\
\hline 3 & 3 & $135-295-387$ & 4 \\
\hline 4 & 4 & $150-380$ & 4 \\
\hline 5 & 5 & $191-192-336-352$ & 12 \\
\hline 6 & $6-13$ & $57-274-386-395$ & 7 \\
\hline 7 & $7-16-19-26-31$ & $0-70-80-86-103-141-178-180-235-255$ & 14 \\
\hline 8 & $8-9-11-12-17-22-24-25-28-32$ & $\begin{array}{l}0-68-70-85-86-90-93-108-130-139-140- \\
141-143-155-178-180-218-235-242-255-\end{array}$ & \\
\hline & & $325-355-382-385-404$ & 21 \\
\hline 9 & 10 & 233-302-362 & 3 \\
\hline 10 & 14 & $139-290-291-340$ & 7 \\
\hline 11 & 15 & $74-78-300$ & 5 \\
\hline 12 & $20-21$ & $122-129-153-197-286-309$ & 7 \\
\hline 13 & 23 & $18-356-365-377-419$ & 9 \\
\hline 14 & 27 & $86-90-108$ & 4 \\
\hline 15 & $29-30$ & $16-72-286-398$ & 10 \\
\hline 16 & 33 & $151-340-366$ & 6 \\
\hline 17 & $34-35$ & 44-46-53 & 23 \\
\hline
\end{tabular}




\section{TABLA 4}

Áreas de consenso (AC) obtenidas usando grillas de $1^{\circ} \mathrm{x} 1^{\circ}$. Para cada $\mathrm{AC}$ se indica: áreas individuales (AI) incluidas, especies endémicas presentes tamaño del área (expresado en número de celdas).

Consensus areas (AC) obtained using grids of $1^{\circ} \times 1^{\circ}$. Included individual areas (AI), endemic species, area size (expressed as cell numbers).

\begin{tabular}{|c|c|c|c|}
\hline Número de áreas & Áreas individuales incluidas & Especies presentes & $\overline{\Gamma a m a n ̃ o ~ d e l ~ a ́ r e a ~}$ \\
\hline \multirow[t]{3}{*}{1} & $1-5-6-9-63-105$ & $0-67-68-70-80-85-86-87-92-93-99-140-141-143-$ & \\
\hline & & 154-157-160-178-180-211-216-218-234-235-242- & \\
\hline & & $325-372-379-382-385-402-403-404$ & 11 \\
\hline 2 & 2 & $92-98-99-100-157-158-160-238-314$ & 4 \\
\hline \multirow[t]{11}{*}{3} & $3-4-7-13-14-15-16-$ & $1-12-16-17-19-21-27-40-41-42-43-44-45-46-47-$ & \\
\hline & $17-18-21-22-27-28-29-30-$ & $48-49-50-51-52-53-57-58-59-60-61-62-63-65-72-$ & \\
\hline & $34-35-36-40-41-42-43-44-$ & $75-81-91-94-95-112-124-125-127-135-174-194-203-$ & \\
\hline & $45-46-49-50-51-52-53-54-$ & $217-219-223-224-226-227-228-279-280-286-295-$ & \\
\hline & $56-58-61-62-64-65-67-68-$ & $322-334-345-346-347-353-370-381-386-387-394-$ & \\
\hline & $69-71-72-73-74-75-76-77-$ & $395-397-398-400-401-410-416-417-422$ & \\
\hline & $78-79-80-81-82-86-87-88-$ & & \\
\hline & $90-91-92-93-94-95-96-98-$ & & \\
\hline & $99-100-101-102-103-104-106-$ & & \\
\hline & $107-108-112-113-115$ & & \\
\hline & 48 & & \\
\hline 4 & $8-39$ & $16-51-72-122-129-153-197-286-309-353-398$ & 6 \\
\hline 5 & 10 & $18-132-354-356-357-365-377-419$ & 5 \\
\hline \multirow[t]{2}{*}{6} & $11-47-48-60-83-97-111$ & $0-15-75-79-80-83-86-91-99-133-141-211-217-$ & \\
\hline & & $253-254-276-322-359-372-384-403-412$ & 17 \\
\hline 7 & $12-20-23-24$ & $12-13-19-44-63-72-74-78-81-226-300$ & 11 \\
\hline 8 & $19-70-89$ & $67-68-71-76-85-87-143-154-221-242-325-332-402$ & 9 \\
\hline 9 & 25 & $35-101-244-248-407$ & 9 \\
\hline 10 & 26 & $35-101-244-294-308-339$ & 7 \\
\hline 11 & 31 & $132-150-354-376-380-391$ & 7 \\
\hline \multirow[t]{2}{*}{12} & 32 & $186-191-192-233-302-304-305-306-328-336-352-$ & \\
\hline & & $361-362-364$ & 5 \\
\hline 13 & $33-55-57$ & $3-66-193-299-340-343-366-389-424$ & 10 \\
\hline 14 & $37-38$ & $3-292-299-343$ & 9 \\
\hline 15 & 59 & $67-83-141-211-216-402-403$ & 8 \\
\hline 16 & 66 & $43-46-53-236-401$ & 7 \\
\hline 17 & 84 & $219-241-252-253$ & 11 \\
\hline 18 & 85-109 & $65-202-224-416-421$ & 17 \\
\hline 19 & 110 & $42-45-58-416$ & 19 \\
\hline 20 & $114-116$ & $41-275-277-399$ & 38 \\
\hline
\end{tabular}


(A)
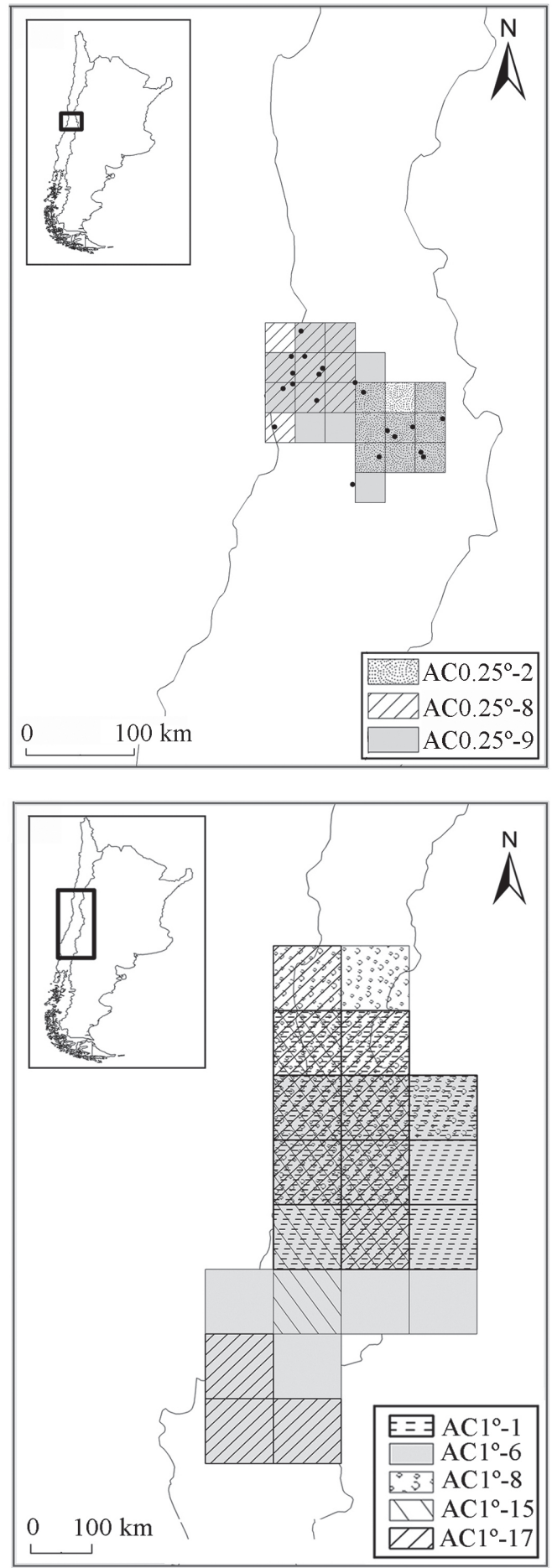

(C)
(B)
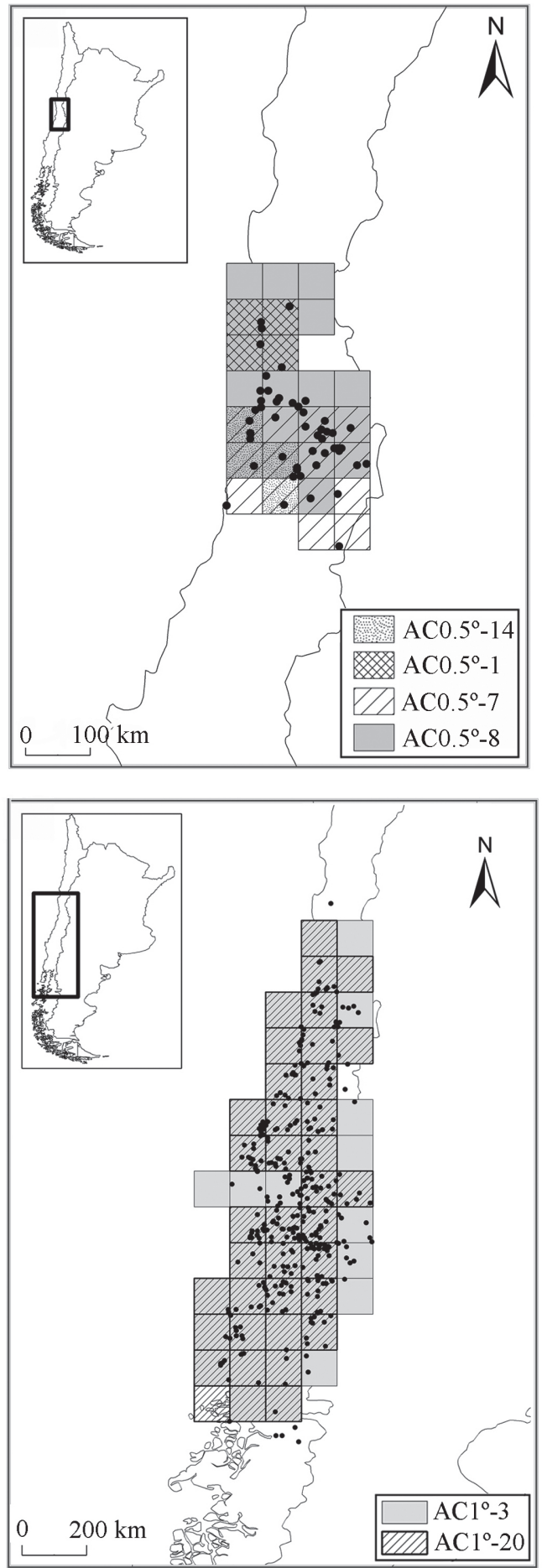

(D)

Fig. 1: Áreas de consenso (AC) correspondientes a la subregión chilena central obtenidas con grillas de $0,25^{\circ} \times 0,25^{\circ}(\mathrm{A}), 0,5^{\circ} \times 0,5^{\circ}(\mathrm{B})$, y $1^{\circ} \times 1^{\circ}(\mathrm{C}-\mathrm{D})$.

Consensus areas (AC) corresponding to central Chilean subregion, obtained with grids of $0.25^{\circ} \times 0.25^{\circ}(\mathrm{A}), 0.5^{\circ} \times 0.5^{\circ}(\mathrm{B})$, and $1^{\circ} \times 1^{\circ}(\mathrm{C}-\mathrm{D})$. 


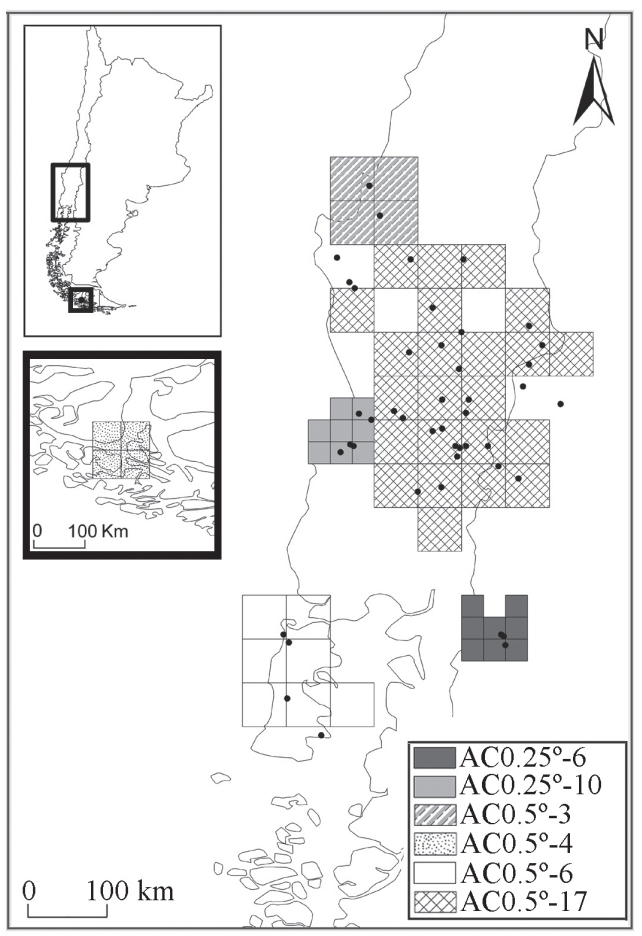

(A)

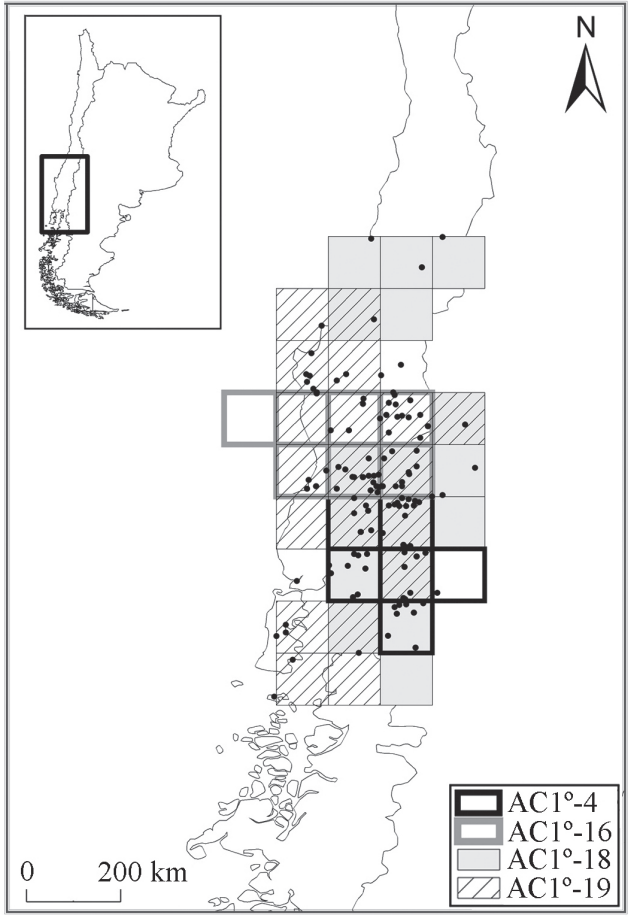

(B)

Fig. 2: Áreas de consenso (AC) correspondientes a la subregión Subantártica obtenidas con grillas de $0,25^{\circ} \times 0,25^{\circ}(\mathrm{A}), 0,5^{\circ} \times 0.5^{\circ}(\mathrm{B})$, y $1^{\circ} \times 1^{\circ}(\mathrm{C})$.

Consensus areas (AC) corresponding to Subantartic subregion obtained with grids of $0.5^{\circ} \times 0.5^{\circ}(\mathrm{A}), 0.5^{\circ} \times 0.5^{\circ}(\mathrm{B})$, and $1^{\circ} \times 1^{\circ}(\mathrm{C})$.

En el análisis de $0,5^{\circ}$ las provincias biogeográficas de Maule y bosque valdiviano se reconocen como una unidad en el $\mathrm{AC}_{0,5} 17$ (Fig. 2A) aunque pequeños fragmentos de estas provincias son reconocidos también como unidades separadas: $\mathrm{AC}_{0,5} 3$ corresponde a un sector de la provincia del Maule (Fig. 2A), y $\mathrm{AC}_{0,5} 6$ a parte del bosque valdiviano. En esta escala de análisis el Archipiélago de Juan Fernández no se recupera en forma completa, si bien las islas que lo componen se identifican como áreas de endemismo independientes definidas por conjuntos de especies endémicas propios (Isla Alejandro Selkirk el $\mathrm{AC}_{0,5}$ 9; Isla Robinson Crusoe: $\mathrm{AC}_{0,5}$ 5. (Fig. 3B, Tabla 3). El uso de este tamaño de grilla permitió reconocer el área más austral de nuestro análisis, AC 0,5 4 (Fig. 2A), caracterizada por la presencia de Peryphus rufoplagiatus y Trechisibus stricticollis (spp: 380 y 150) como especies endémicas.

Al usar grillas de $1^{\circ} \times 1^{\circ}$ las provincias de bosque valdiviano y Maule se reconocen claramente como unidades diferentes $\left(\mathrm{AC}_{1} 19 \mathrm{y}\right.$ $\mathrm{AC}_{1}$ 16, Fig. 2B, Tabla 4). $\mathrm{AC}_{1} 19$ corresponde al bosque valdiviano e incluye un área de menor tamaño caracterizada por un conjunto de especies diferente $\left(\mathrm{AC}_{1} 4\right)$, coincidente con una pequeña fracción de la tundra magallánica $\mathrm{O}$ páramo magallánico (Morrone 2001a, Fig. 2B), mientras $\mathrm{AC}_{1} 16$ recupera la provincia de Maule (Morrone 2001a). Sin embargo, de manera similar a lo ocurrido en los análisis de $0,5^{\circ}$, en esta escala también se recupera un área que incluye fracciones de bosque valdiviano y Maule $\left(\mathrm{AC}_{1}\right.$ 18). El Archipiélago de Juan Fernández también es identificado con este tamaño de celda $\left(\mathrm{AC}_{1} 12\right)$, aunque sus islas no son recuperadas como unidades separadas (Fig. 3C).

\section{Subregión Patagónica}

En los análisis de $0,25^{\circ}$ no se identificó ningún área dentro de la subregión Patagónica; mientras que usando grillas de $0,5^{\circ}$ se reconocieron cuatro $\mathrm{AC}$, dos de ellas 
(A)

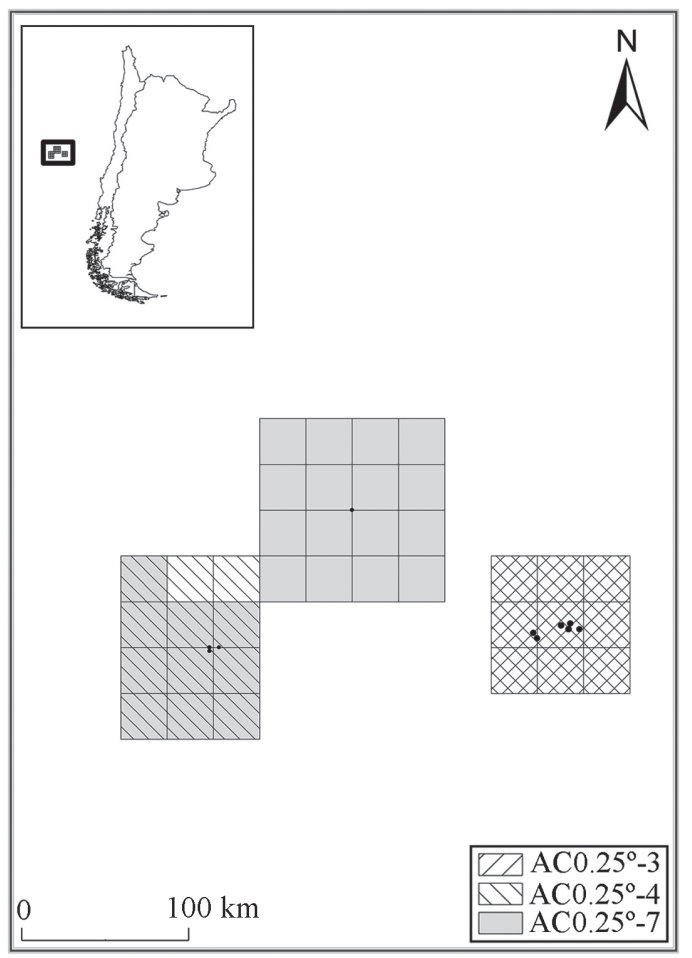

(B)

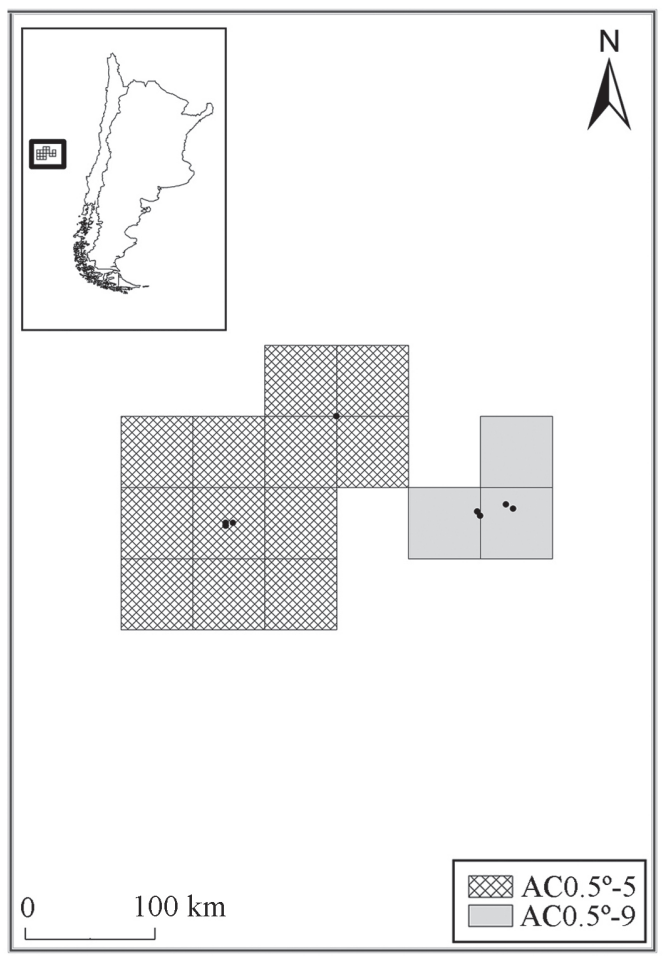

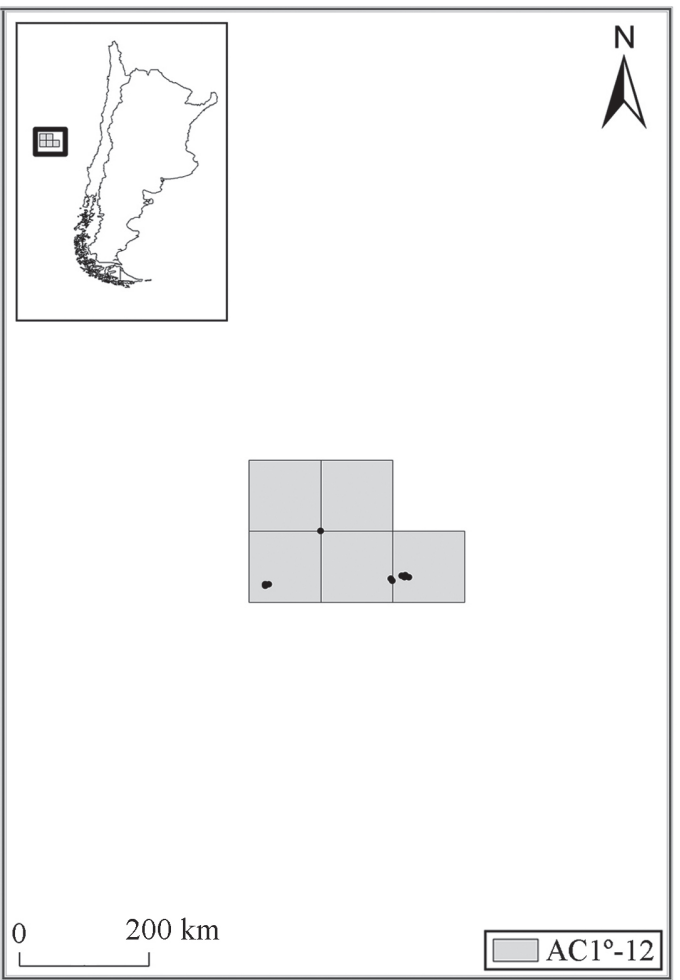

(C)

Fig. 3: Áreas de consenso (AC) correspondientes a la provincia de las islas Juan Fernández (subregión Subantártica) obtenidas con grillas de $0,25^{\circ}$ x $0,25^{\circ}(\mathrm{A}), 0,5^{\circ} \times 0,5^{\circ}(\mathrm{B})$, y $1^{\circ} \times 1^{\circ}(\mathrm{C})$.

Consensus areas (AC) corresponding to the province of Juan Fernandez Islands, included in the Subantartic subregion obtained with grids of $0.25^{\circ} \times 0.25^{\circ}(\mathrm{A}), 0.5^{\circ} \times 0.5^{\circ}(\mathrm{B})$, and $1^{\circ} \times 1^{\circ}(\mathrm{C})$. 


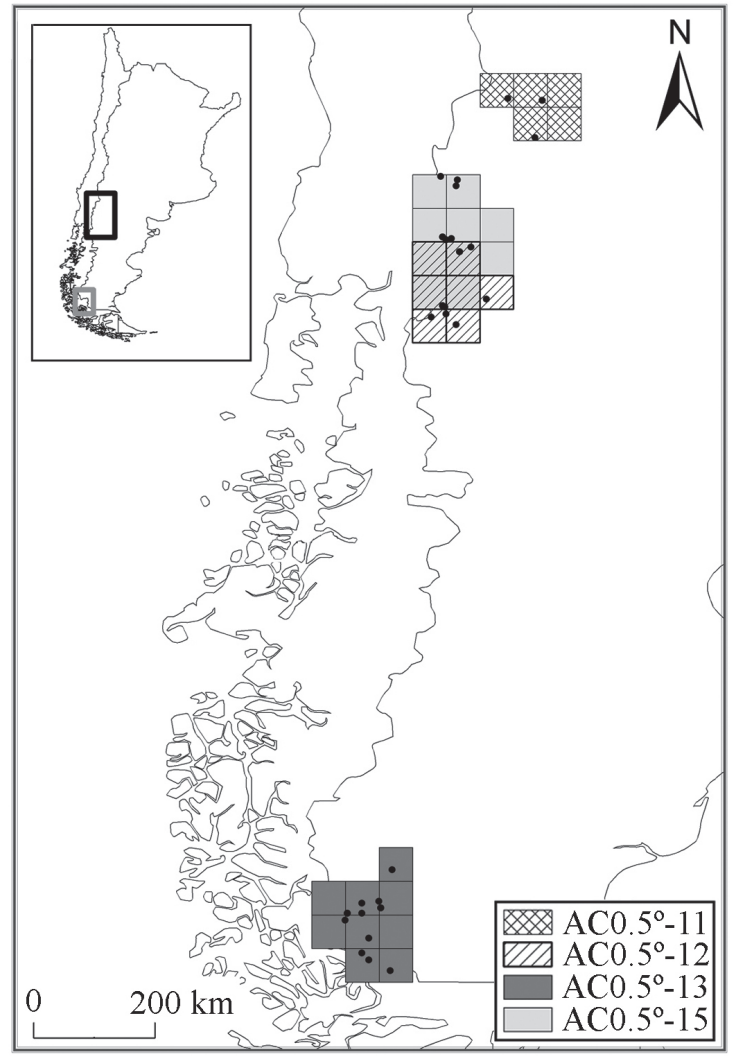

(A)

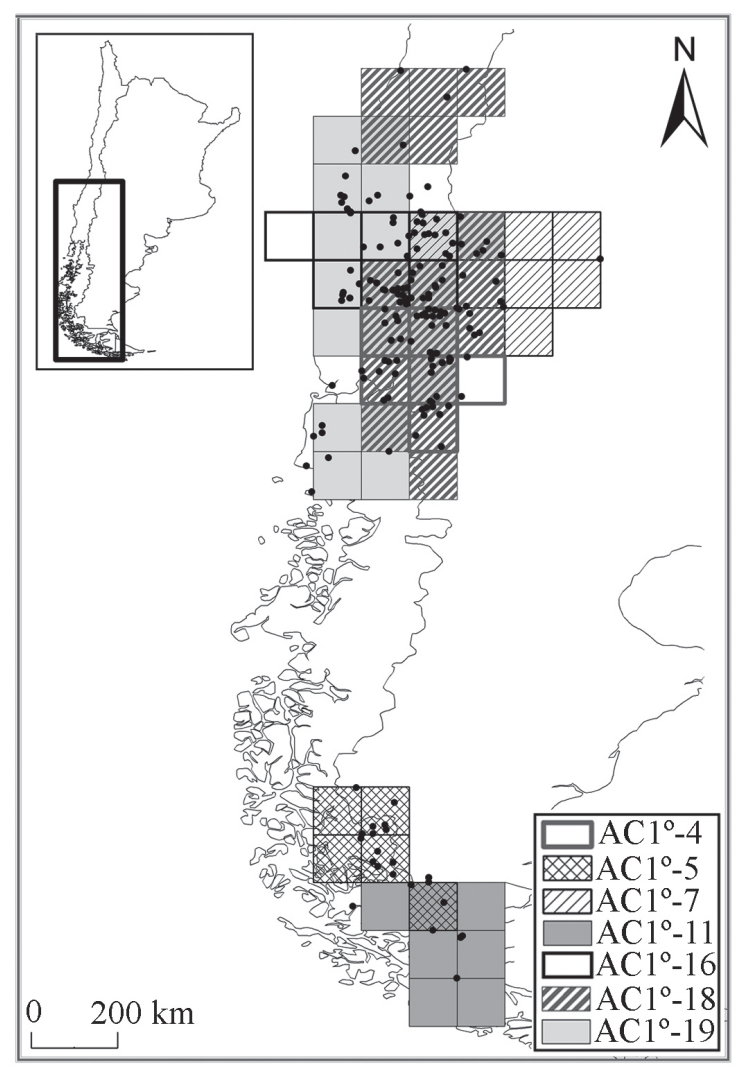

(B)

Fig. 4: Áreas de consenso (AC) correspondientes a la subregión patagónica obtenidas con grillas de $0,5^{\circ} \times 0,5^{\circ}(\mathrm{A}), \mathrm{y} 1^{\circ} \times 1^{\circ}(\mathrm{B})$.

Consensus areas (AC) corresponding to Patagonian subregion obtained with grids of $0,5^{\circ} \times 0,5^{\circ}(\mathrm{A})$, and $1^{\circ} \times 1^{\circ}(\mathrm{B})$.

parcialmente superpuestas $\left(\mathrm{AC}_{0,5} 11, \mathrm{AC}_{0,5} 12\right.$, $\mathrm{AC}_{0,5} 13$ y $\mathrm{AC}_{0,5} 15$, Fig. 4A). El $\mathrm{AC}_{0,5} 11$, definida por tres especies endémicas, corresponde a la provincia biogeográfica de Patagonia central (Morrone 2001b), específicamente a la región austral del distrito de la Payunia (Morrone et al. 2002, Domínguez et al. 2006). $\mathrm{AC}_{0,5} 12$ está ubicada en la región septentrional de la provincia de Patagonia subandina (Fig. 4A), y $\mathrm{AC}_{0,5} 15$ ocupa la región del distrito de la Patagonia occidental (Domínguez et al. 2006). El $\mathrm{AC}_{0,5} 13$ coincide con el área denominada por Domínguez et al. (2006) como Patagonia subandina austral. En los análisis de $1^{\circ}$ la estepa patagónica queda definida por $\mathrm{AC}_{1} 5, \mathrm{AC}_{1} 7$ y $\mathrm{AC}_{1} 11$ (Fig. 4B). $\mathrm{AC}_{1} 5$ corresponde a la subandina austral. $\mathrm{AC}_{1}$ 7 representa la región austral del distrito de Payunia (Domínguez et al. 2006), y si bien se superpone espacialmente con otras $\mathrm{AC}\left(\mathrm{AC}_{1} 4\right.$,
$\mathrm{AC}_{1} 16, \mathrm{AC}_{1} 18$ y $\mathrm{AC}_{1}$ 19), $\mathrm{AC}_{1} 7$ posee un conjunto característico de especies endémicas que le otorga identidad como área de endemismo (spp: 12-13-19-44-63-72-74-78-81226-300, Anexo 1). Finalmente, $\mathrm{AC}_{1} 11$ corresponde al distrito de Patagonia austral de la provincia patagónica central (Roig-Juñent et al. 1994, Domínguez et al. 2006).

\section{Zona de transición sudamericana}

En los análisis de $0,25^{\circ}$ se identificaron dos AC dentro de la zona de transición sudamericana (Morrone 2006, Fig. 5A). $\mathrm{AC}_{0,25} 1$ se ubica en la región austral de la provincia biogeográfica de la Puna, coincidiendo con la región denominada Puna Cuyana (Martínez Carretero 1995), y $\mathrm{AC}_{0,25} 5$ ubicada al noroeste de Argentina, corresponde a la Puna Jujeña (Martínez Carretero 1995), región caracterizada 
(A)
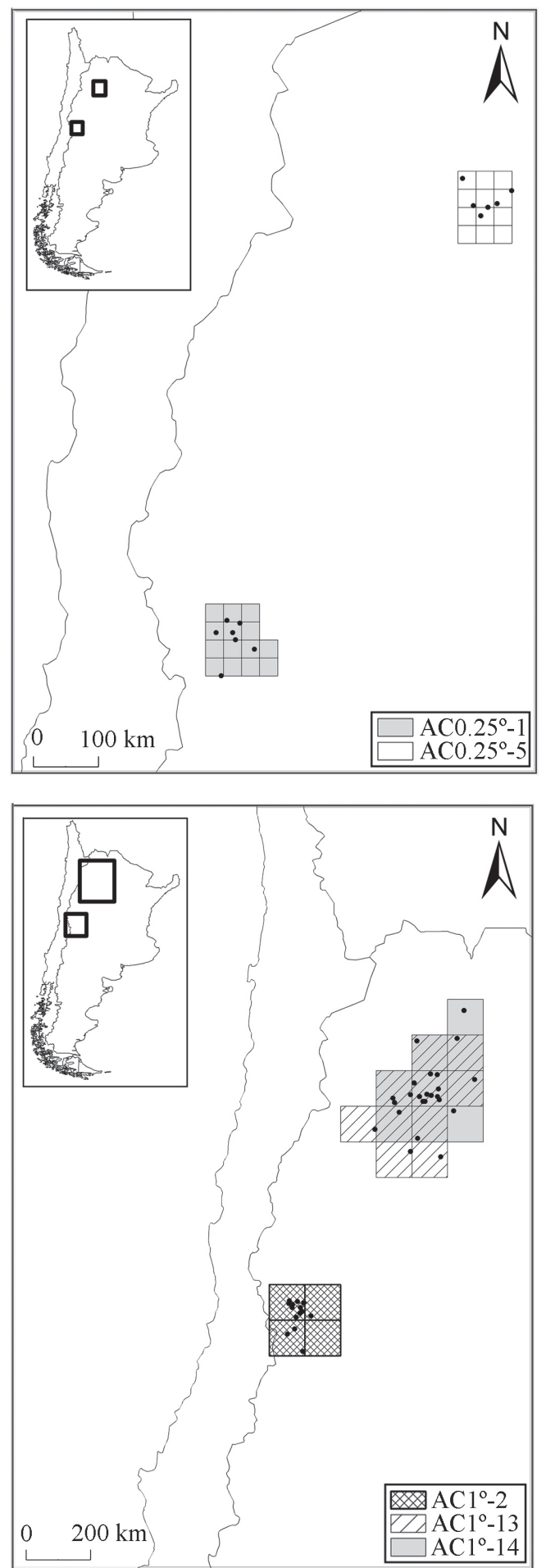

(C)
(B)

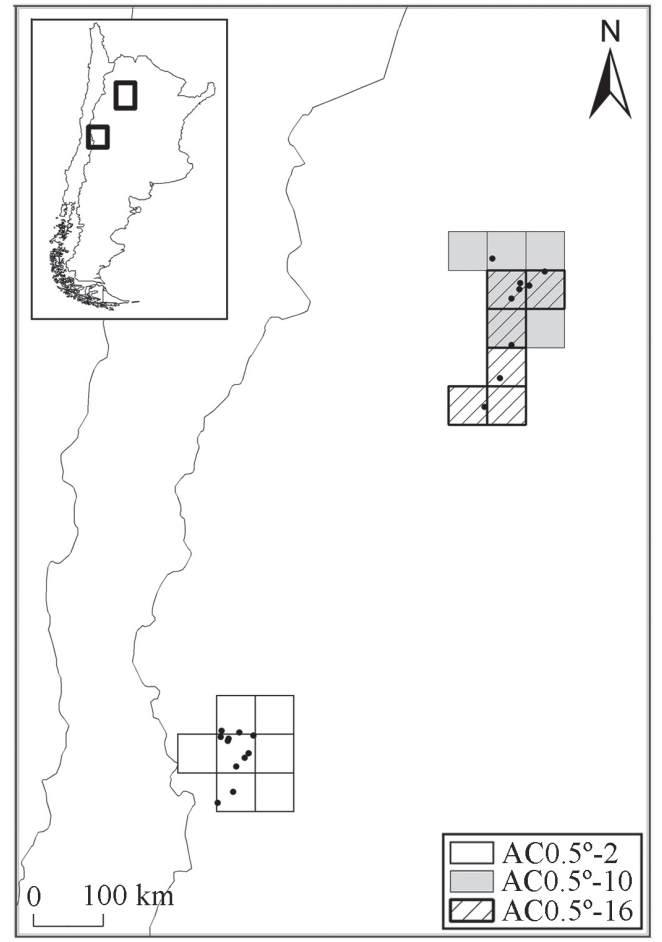


por la presencia de pastizales de altura. A pesar de ser una de las áreas más septentrionales de América del Sur austral, la Puna jujeña presenta una fauna rica en elementos australes (Roig et al. en prensa).

Al igual que en los análisis de $0,25^{\circ}$, las matrices de $0,5^{\circ}$ identifican áreas de endemismo dentro de la región de la Puna argentina (Morrone 2001a, Fig. 5B), coincidentes con los pastizales de altura de la Puna jujeña $\left(\mathrm{AC}_{0,5} 10\right.$ y $\left.\mathrm{AC}_{0,5} 16\right)$ y de la Puna cuyana $\left(\mathrm{AC}_{0,5} 2\right.$; Martínez-Carretero 1995).
La Puna jujeña (Martínez-Carretero 1995) queda representada en los análisis de $1^{\circ}$ por $\mathrm{AC}_{1} 13$ y $\mathrm{AC}_{1} 14$ (Fig. 5C). Estas $\mathrm{AC}$, ubicadas en los sistemas montañosos del noroeste de Argentina, se superponen ampliamente y comparten gran parte de sus especies endémicas, por lo que las consideramos una única área. La región de Puna cuyana (Martínez-Carretero 1995) es recuperada como un área de endemismo por $\mathrm{AC}_{1} 2$, situada en al zona precordillerana (Fig. $5 \mathrm{C})$.

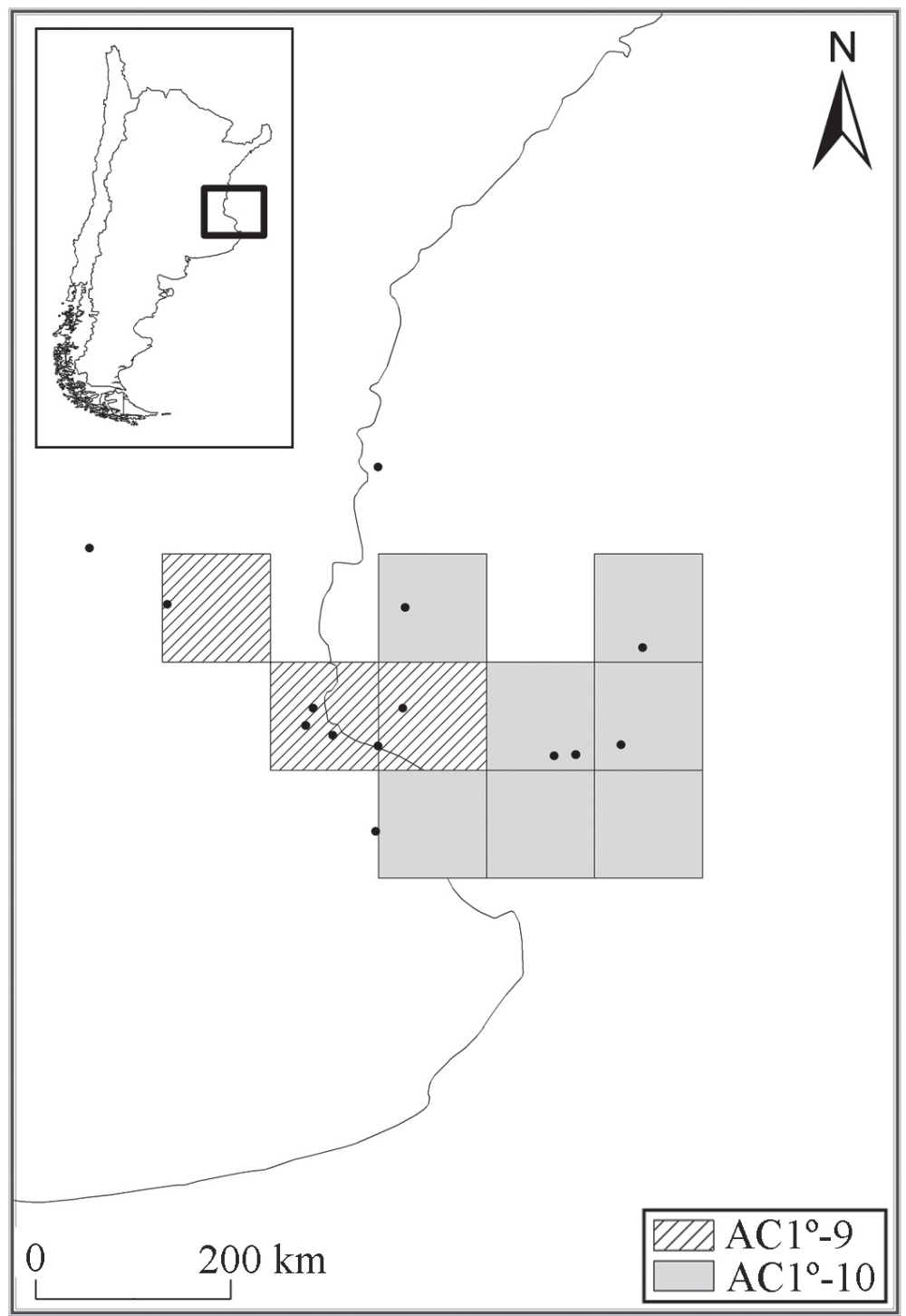

Fig. 6: Áreas de consenso (AC) obtenidas con grillas de $1^{\circ}$ x $1^{\circ}$, que representan sistemas pampeanos.

Consensus areas (AC) obtained with grids of $1^{\circ} \times 1^{\circ}$, that represent pampean systems. 


\section{Sistemas pampeanos}

Además de las áreas de endemismo identificadas dentro de la región andina, en los análisis de $1^{\circ} \times 1^{\circ}$ se recuperaron áreas de endemismo dentro de la región neotropical (Morrone 2001a). $\mathrm{AC}_{1} 9$ y $\mathrm{AC}_{1} 10$ definen parches de la provincia biogeográfica de la Pampa, perteneciente a la subregión chaqueña (Morrone 2001a, Fig. 6). Si bien esta provincia no se considera parte de la región austral de América del Sur (Ringuelet 1961), es vinculada a esta región a través de ciertos sistemas montañosos -como el de Tandilia, Ventania y sur de Uruguay- en los cuales se encuentran numerosos elementos patagónicos (Roig et al. en prensa).

\section{Análisis a nivel de género}

En los análisis a nivel de género solo se reconocieron áreas de endemismo en las matrices de mayor tamaño de celda; identificándose en este caso tres AI que se

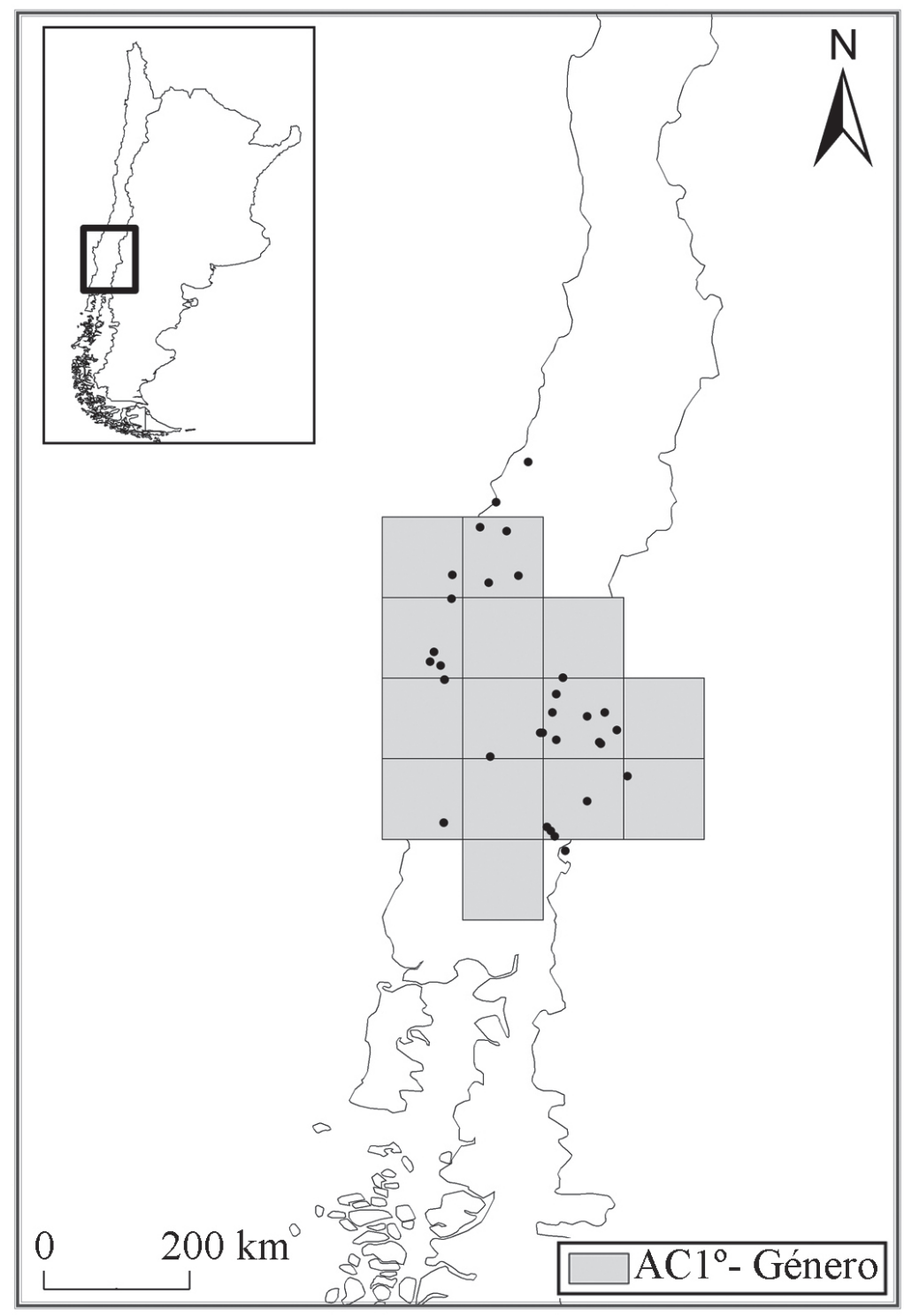

Fig. 7: Áreas de consenso (AC) obtenidas del análisis a nivel de género, usando grillas de $1^{\circ} \mathrm{x} 1^{\circ}$. Consensus areas (AC) obtained from genus-level analysis, using grids of $1^{\circ} \times 1^{\circ}$. 
reúnen en una única $A C$ que abarca las provincias de Maule y Valdiviana, caracterizada por los géneros Nothobroscus, Nemaglossa, Mimophilorhizus, Castascopellus y Lebia (Fig. 7). Estos géneros son característicos de los bosques de Nothofagus, siendo la mayoría endémicos de esta región, ubicada entre los $36^{\circ}$ y $40^{\circ} \mathrm{S}$ y $74^{\circ}-70^{\circ} \mathrm{O}$. Cabe destacar, que si bien Lebia es un género ampliamente distribuido en la región neotropical, dentro de América del Sur austral el género se encuentra representado por una única especie, Lebia azurea, cuya distribución está restringida a los bosques de Nothofagus, y que es la única especie del género incluida en este análisis.

Variación de tamaño de grilla y valores de llenado (R.fill)

El número de áreas de endemismo aumenta con el aumento en el tamaño de celda analizado; el número de $\mathrm{AI}$ se incrementa más de dos veces al pasar de celdas de $0,25^{\circ}$ a celdas de $0,5^{\circ}$ y tres veces al pasar de celdas de $0,5^{\circ}$ a $1^{\circ}$, mientras el número de $\mathrm{AC}$ aumenta en menor proporción, encontrándose $10 \mathrm{AC}$ para celdas de $0,25^{\circ}, 17$ para celdas de $0,5^{\circ}$, y 20 AC para celdas de $1^{\circ}$. (Fig. 8A). De la misma manera, se reconoce un mayor número de especies endémicas a medida que aumentamos el tamaño de celda empleado: 51 especies endémicas son reconocidas al usar una grilla de $0,25^{\circ}, 74$ usando la grilla de $0,5^{\circ}$, y 187 para la grilla de $1^{\circ}$ (Fig. 8B). Algunas especies se identifican como endémicas solo en un tamaño de celda particular. Estas especies "únicas" resultan interesantes, ya que podrían indicar la existencia de patrones característicos de una escala determinada, que permanecerían ocultos al usar otras escalas espaciales. El número de especies únicas también muestra una relación positiva con el aumento de tamaño de celda analizado (Fig. 8B), aunque la cantidad de especies únicas presentes en el análisis de $0,5^{\circ}$ es menor que en los otros casos.

En el caso analizado se observa una tendencia general al aumento del número de áreas de endemismo y de especies endémicas según aumenta el valor de R. fill, si bien la forma en que esto ocurre muestra variaciones entre los diferentes tamaños de grilla (Fig. 9). En los análisis de $0,25^{\circ}$ el número de áreas y de especies endémicas aumenta continuamente

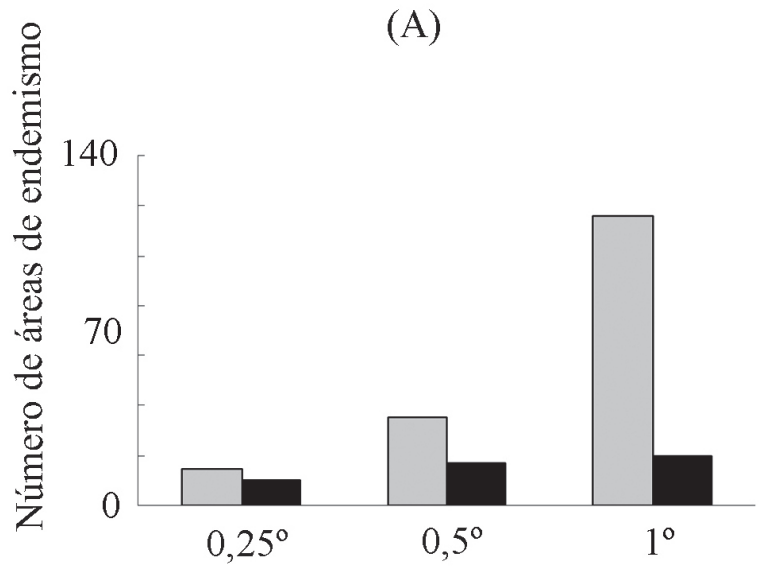

Tamaño de grilla

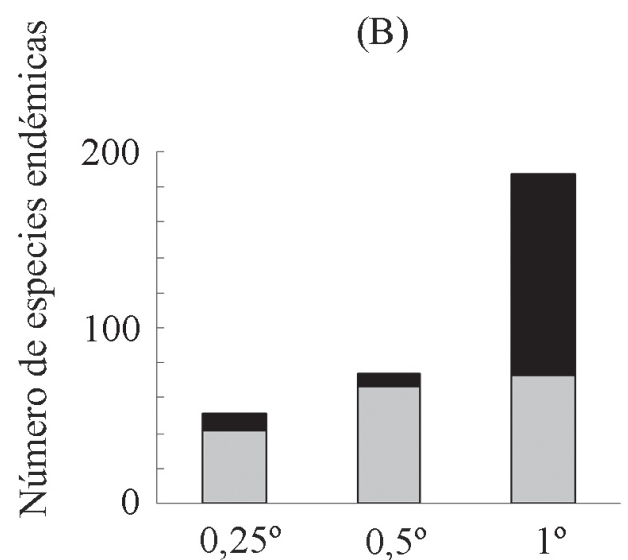

Tamaño de grilla

Fig. 8: Variación de tamaño de grilla: (A) Número de áreas de endemismo encontradas bajo los diferentes tamaños de grilla. La barra gris indica el número de áreas de endemismo individuales (AI), y la barra negra indica el número de áreas de consenso (AC). (B) Número de especies endémicas encontradas bajo diferentes tamaños de grilla. La fracción de la barra rellena en negro indica la proporción de especies endémicas únicas.

Variation between different grid sizes: (A) Number of endemism areas found under the different grid sizes. Grey bars show the number of individual endemism areas (AI), while black bars indicate number of consensus areas (AC). (B) Number of endemic species found under different grid sizes. Black portion of the bar shows the proportion of unique species. 
hasta R. fill 40-50, desde donde comienzan a descender tanto el número de áreas como de especies endémicas hasta R. fill 70-80 donde comienzan a aumentar nuevamente. Ambos valores alcanzan su máximo en R. fill 100. En los tamaños de celda mayores $\left(0,5^{\circ}\right.$ y $\left.1^{\circ}\right)$ los valores máximos de especies endémicas y de áreas de endemismo se encuentran entre valores de R. fill 70-90, siendo menores los valores reportados en R. fill 100 (Fig. 9).

(A)

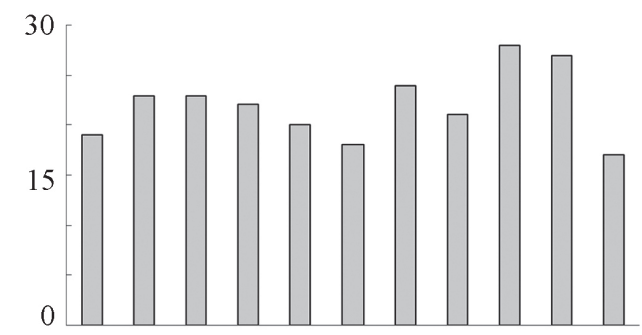

Grilla de $0,25^{\circ}$
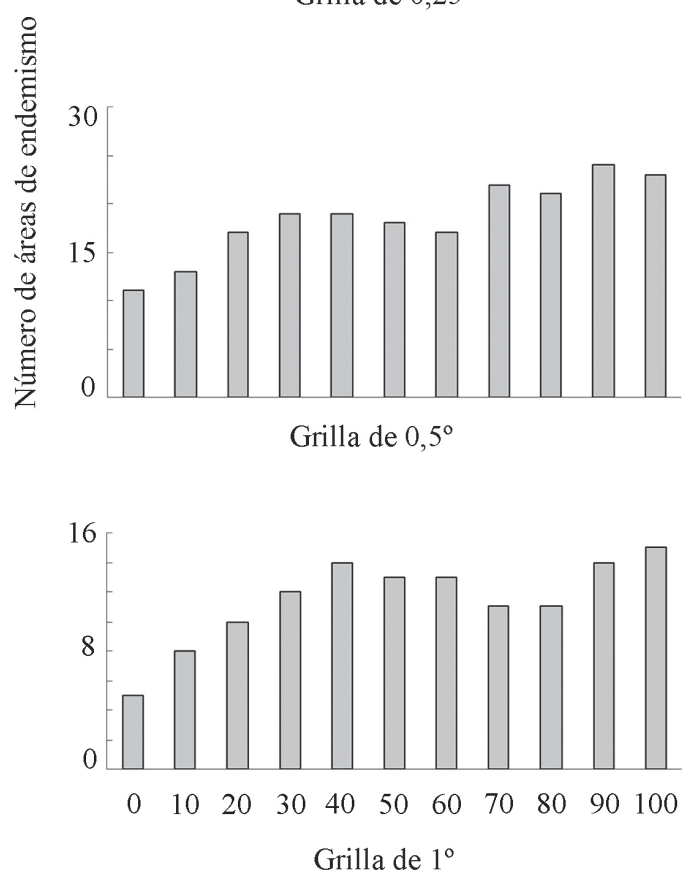

\section{DISCUSIÓN}

Si bien las colecciones biológicas son la fuente de información más importante disponible para la realización de estudios de biodiversidad, muchas veces los datos obtenidos a partir de estas suelen presentar sesgos de muestreo que producen diversos grados de error en la determinación de patrones biogeográficos (Escalante 2005,

(B)

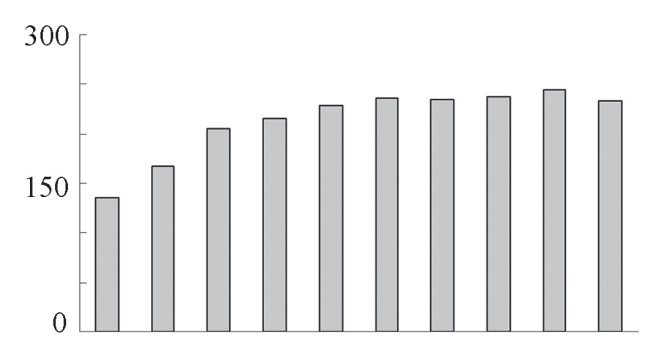

Grilla de $0,25^{\circ}$
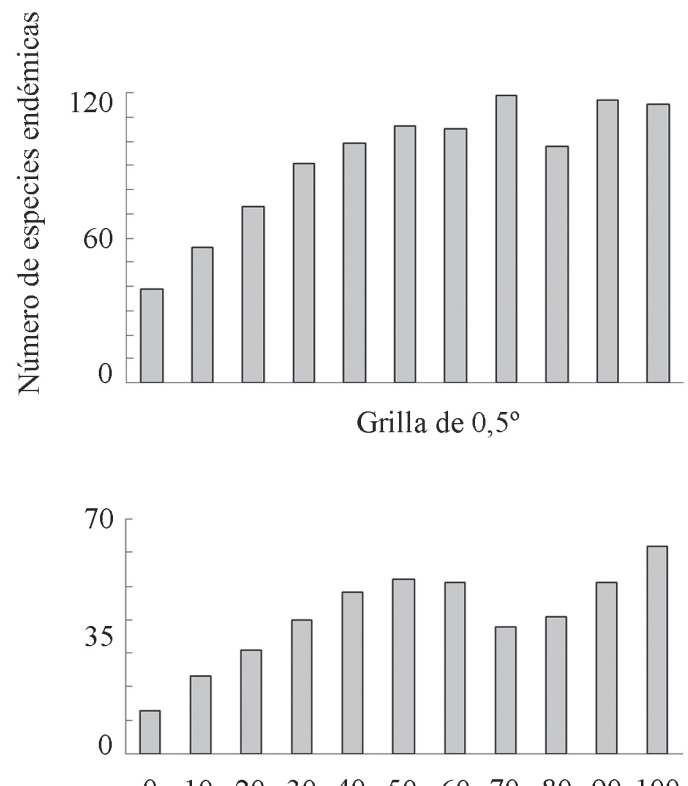

Grilla de $1^{\circ}$

R. fill

Fig. 9: Variación entre diferentes valores de Radio de llenado (R. fill): (A) Número total de especies endémicas encontrado usando diferentes valores de R. fill, y bajo los diferentes tamaños de grilla usados. (B) Número total de áreas de endemismo encontradas usando diferentes valores de R. fill, y bajo los diferentes tamaños de grilla usados.

Variation between different values of Radious of Fill (R. fill): (A) Total number of endemic species found using different R. fill values, and under different grid sizes. (B) Total number of areas of endemism found using different R. fill values, and under different grid sizes. 
Hortal et al. 2007). Los métodos usados en la descripción de áreas de endemismo, así como de otros patrones de distribución (e.g., centros de riqueza y diversidad de especies) son especialmente sensibles a la ausencia de datos en sectores de la región de estudio, debido a que la discontinuidad espacial producida por las ausencias disminuye la probabilidad de simpatría entre especies, modificando así el número, forma, y composición de patrones encontrados. Al mismo tiempo, la ausencia de datos afectará en mayor o menor medida la delimitación de patrones según el tamaño de celda empleado: mientras el uso de celdas pequeñas acentúa la discontinuidad espacial (cuanto más pequeñas, mayor será el numero de celdas con ausencia de información), el empleo de celdas grandes aumenta artificialmente el rango de distribución de las especies, pudiendo eliminar por completo la discontinuidad en caso de que la celda utilizada sea demasiado grande.

Muchas veces la ausencia de datos en determinados sitios (ciudades, sitios inaccesibles, etc.) responde a falta de muestreo. Por lo que el sesgo muestral es un factor que debe ser considerado al momento de definir estrategias de análisis apropiadas, así como durante la interpretación de los patrones encontrados.

En el caso aquí presentado la discontinuidad espacial de los datos fue tratada mediante un procedimiento heurístico, asignando distribuciones potenciales para las especies según la función de llenado (R. fill) implementada en NDM/VNDM. R. fill asigna presencias potenciales de especies siguiendo un principio matemático simple basado en distancia geográficas (Szumik \& Goloboff 2004), de manera muy diferente a la complejidad matemática implementada en los métodos de predicción de especies siguiendo modelos de nicho ecológico (Austin 2002, Zaniewski et al. 2002, Phillips et al. 2006). Lejos de pretender remplazar estos modelos, el uso de $\mathrm{R}$. fill proporciona una alternativa sencilla para aproximar presencias potenciales de especies usando solo los datos de distribución observados. Este "llenado" de especies se realiza desde NDM/VNDM en un paso previo a la búsqueda de patrones, y el tiempo que esta operación consume es mínimo.
Los datos tratados presentan gran heterogeneidad en el número de registros de ocurrencia incluidos por especie $(43 \%$ especies poseen más de cinco registros, $57 \%$ el resto posee una cantidad menor), y en algunos casos, estos registros son muy distantes entre sí. Al ser mapeados en grillas, las distribuciones producidas por estos datos aparecen "fragmentadas" y la aplicación de la función de llenado (R. fill) permite completarlas, resolviendo parcialmente la discontinuidad espacial.

En el caso analizado la aplicación de esta función aumenta el número de áreas y especies endémicas encontradas. El valor de R. fill en el cual se encuentran mayor número de áreas de endemismo y especies endémicas varía de acuerdo al tamaño de celda empleado, lo que señala cierta dependencia entre la función y el tamaño de celda.

La forma en que distintos valores de esta función afectan la determinación de patrones depende principalmente de la estructura espacial de los datos analizados, por lo que no es posible generalizar conclusiones acerca de la incidencia de esta función a partir del estudio de un único caso. Análisis comparativos entre conjuntos de datos con diversas características (i.e., diferentes números de registros, datos con diferentes tipos de distribución) pueden contribuir a alcanzar una mejor comprensión sobre el comportamiento de esta función y su implicancia en la determinación de patrones biogeográficos.

Por otro lado, la búsqueda de patrones biogeográficos se ve fuertemente afectada por la dependencia de las metodologías de análisis al uso de grillas. El empleo de distintas escalas espaciales (i.e., tamaño de celda) resulta generalmente en la identificación de diferentes patrones y, como fue mencionado, esta variación se ve a su vez afectada por las características particulares de los datos usados (e.g., distribución espacial de los datos). Aunque algunos autores han analizado los efectos de la escala de análisis en la identificación de áreas de endemismo (Morrone \& Escalante 2002, Laffan \& Crisp 2003), este tema ha sido abordado principalmente desde el marco de la biogeografía ecológica (Levin 1992, Anderson \& Marcus 1993, Willig et al. 2003); y a pesar de ser un factor determinante en la descripción 
de áreas de endemismo en la mayoría de los trabajos aplicados se considera una única escala espacial (e.g., Roig-Juñent et al. 2002, Pizarro-Araya \& Jerez 2004). Si bien la complejidad del problema sugiere que debemos ser cuidadosos a la hora de generalizar a partir de un caso de estudio, los resultados del presente análisis nos permiten describir algunos efectos de la variación de escala en la delimitación de áreas de endemismo. En general, el reconocimiento de áreas pequeñas y de áreas disyuntas resulta encubierto al usar celdas de gran tamaño, siendo identificadas frecuentemente al usar celdas pequeñas (e.g., algunas subáreas de la provincia de Santiago solo fueron reconocidas en los análisis de $0,25^{\circ}$ y $0,50^{\circ}$; las islas del Archipiélago de Juan Fernández solo se recuperan en los dos tamaños menores). Al mismo tiempo, al usar celdas pequeñas aumenta la discontinuidad espacial de los datos, disminuyendo generalmente la probabilidad de simpatría entre especies y por lo tanto el número de patrones recuperados. Por esto, el uso de celdas pequeñas resulta especialmente inadecuado al analizar poca cantidad de datos, o bien cuando estos se encuentran muy dispersos espacialmente. Al contrario, el uso de celdas grandes produce diversos grados de "aglutinamiento" en los datos, lo que dificulta el reconocimiento de áreas de endemismo de extensión reducida. Este tipo de celdas puede representar, sin embargo, una ventaja en el reconocimiento de unidades continuas de gran extensión que bajo escalas menores aparecen fragmentadas, o bien no se reconocen.

Muchas de las regionalizaciones biogeográficas usadas para la comparación de nuestros resultados fueron obtenidas mediante métodos distintos del empleado en nuestro análisis; incluso en algunos casos, estas regionalizaciones se realizaron sobre la base del conocimiento natural de los autores, prescindiendo para ello de un protocolo formal (Cabrera \& Willink 1973). A pesar de las diferencias metodológicas mencionadas, gran parte de las áreas de endemismo obtenidas en este análisis corresponden total o parcialmente a alguna de las biorregiones descritas con anterioridad. Además de indicar coherencia biológica en los resultados encontrados, este hecho señala gran consistencia entre la teoría biológica que sustenta el método usado (AE) y su implementación computacional (NDM/ VNDM, Goloboff 2004).

La información analizada (3.989 registros) produjo el reconocimiento de numerosas áreas de endemismo dentro de América del Sur austral, permitiendo la delimitación de un esquema biogeográfico general de la región. A través de todas las escalas de análisis se reconocieron áreas de endemismo equivalentes a regiones descritas como "naturales" por diferentes autores (Cabrera \& Willink 1973, Roig-Juñent 1994, Martínez-Carretero 1995, Morrone 2001a, 2001b, Roig-Juñent \& Flores 2001, Morrone et al. 2002, Domínguez et al. 2006, Tabla 5), así como un centro de diversidad mundial (hotspot) (provincia de Chile central, Myers 2000). Ciertas unidades biogeográficas -provincias de Santiago, Archipiélago de Juan Fernández, Maule, bosque valdiviano, y distritos de Puna jujeña y Puna cuyana- se reconocieron consistentemente bajo diferentes escalas espaciales, lo que sugiere que estos patrones son unidades naturales robustas.

Por otro lado, si bien algunas áreas de endemismo encontradas coinciden en forma más o menos completa con regiones biogeográficas previamente definidas, otras identifican subáreas dentro de estas regiones, o bien conforman áreas de mayor tamaño fusionando dos o más regiones naturales. En contraste con la visión tradicional de áreas de endemismo, caracterizada por la necesaria homogeneidad en cada unidad y la rigidez de los límites de distintas áreas, el análisis de endemicidad (Szumik et al. 2002, Szumik \& Goloboff 2004) permite recuperar áreas de endemismo que se extienden a través de diferentes regiones biogeográficas (e.g., $\mathrm{AC}_{0,5}$ 17, $\left.\mathrm{AC}_{1} 3, \mathrm{AC}_{1} 20\right)$, y también que áreas de endemismo vecinas se superpongan espacialmente (e.g., $\mathrm{AC}_{1}$ 7), otorgando una idea más real de cómo ocurren en la naturaleza estos patrones biogeográficos. Esta flexibilidad operativa nos abre una nueva perspectiva en la búsqueda de áreas de endemismo, sugiriéndonos nuevas preguntas que nos encaminan hacia una comprensión más profunda de estos patrones naturales. 


\section{TABLA 5}

Correspondencia entre las áreas de consenso encontradas bajo diferentes tamaños de grilla y áreas naturales descritas por otros autores. Los casos en que el área de consenso es menor al área biogeográfica correspondiente, son señalados con un asterisco $(*)$.

Correspondence between consensus areas founded at different grid size and natural areas described by other authors. Cases in which consensus area is smaller than the corresponding biogeographical region, are marked with an asterisk $(*)$.

\begin{tabular}{|c|c|c|c|c|c|c|c|}
\hline \multirow{2}{*}{$\begin{array}{l}\text { Área biogeográfica } \\
\text { Subregión }\end{array}$} & \multicolumn{7}{|c|}{ Referencia } \\
\hline & Provincia & Distrito & Otro & $\mathrm{CC} 0.25^{\circ}$ & $\mathrm{AC} 0.50^{\circ}$ & $\mathrm{AC} 1^{\circ}$ & \\
\hline \multirow[t]{5}{*}{ Chilena central } & & & & & & $\mathrm{X}$ & Morrone (2001a) \\
\hline & Santiago & & & $\mathrm{X}$ & $X$ & $\mathrm{X}$ & Morrone (2001a) \\
\hline & & & $\begin{array}{l}\text { Cordillera } \\
\text { de la Costa }\end{array}$ & $\mathrm{X}$ & $\mathrm{X}$ & & $\begin{array}{l}\text { Roig-Juñent \& } \\
\text { Flores (2001) }\end{array}$ \\
\hline & & & $\begin{array}{l}\text { Valle central } \\
\text { y andino }\end{array}$ & $X$ & $X$ & & $\begin{array}{l}\text { Roig-Juñent \& } \\
\text { Flores (2001) }\end{array}$ \\
\hline & Coquimbo & & & & $X$ & & $\begin{array}{l}\text { Roig-Juñent (1994), } \\
\text { Morrone (2001a) }\end{array}$ \\
\hline \multirow[t]{12}{*}{ Patagónica } & & & & & & & \\
\hline & \multicolumn{2}{|c|}{ Patagonia central } & & & & & $\begin{array}{l}\text { Morrone (2001a, } \\
\text { 2001b) }\end{array}$ \\
\hline & \multirow{3}{*}{\multicolumn{2}{|c|}{ Payunia }} & & & & & $\begin{array}{l}\text { Cabrera \& Willink } \\
\text { (1973), Morrone } \\
\text { et al. (2002) }\end{array}$ \\
\hline & & & $\begin{array}{l}\text { Payunia } \\
\text { austral }\end{array}$ & & $X$ & $\mathrm{X}$ & $\begin{array}{l}\text { Domínguez et al. } \\
\text { (2006) }\end{array}$ \\
\hline & & & $\begin{array}{l}\text { Payunia } \\
\text { septentrional }\end{array}$ & & & $\mathrm{X}$ & $\begin{array}{l}\text { Domínguez et al. } \\
\text { (2006) }\end{array}$ \\
\hline & & \multirow[t]{3}{*}{ Central } & & & & & Morrone et al. (2002) \\
\hline & & & Santacrucino & & & & $\begin{array}{l}\text { Domínguez et al. } \\
\text { (2006) }\end{array}$ \\
\hline & & & Chubutino & & & & $\begin{array}{l}\text { Domínguez et al. } \\
(2006)\end{array}$ \\
\hline & \multirow[t]{3}{*}{ Subandino } & Fueguino & & & & $X$ & $\begin{array}{l}\text { Morrone et al. (2002) } \\
\text { Morrone (2001a, } \\
\text { 2001b), Morrone } \\
\text { et al. (2002) }\end{array}$ \\
\hline & & & Septentrional & & $X$ & & $\begin{array}{l}\text { Domínguez et al. } \\
(2006)\end{array}$ \\
\hline & & & Meridional & & $\mathrm{X}$ & $\mathrm{X}^{*}$ & $\begin{array}{l}\text { Domínguez et al. } \\
\text { (2006) }\end{array}$ \\
\hline & Occidental & & & & $X$ & $X$ & $\begin{array}{l}\text { Cabrera \& Willink } \\
\text { (1973) Domínguez } \\
\text { et al. (2006) }\end{array}$ \\
\hline
\end{tabular}

Subantártica

\begin{tabular}{|c|c|c|c|}
\hline Islas Juan Fernández & & $X$ & \\
\hline & Isla Robinson & & $X$ \\
\hline & $\begin{array}{l}\text { Crusoe } \\
\text { Isla Alejandro } \\
\text { Selkirk }\end{array}$ & X & $\mathrm{X}$ \\
\hline Maule & & $X^{*}$ & $\mathrm{X}^{*}$ \\
\hline Bosque valdiviano & & $\mathrm{X}^{*}$ & $\mathrm{X}^{*}$ \\
\hline Bosque magallánico & & & \\
\hline Páramo magallánico & & & \\
\hline Islas Malvinas & & & \\
\hline
\end{tabular}

Zona de transición sudamericana (sensu Morrone 2006) o subregión páramo puneña

Puna

Puna Cuyana
Puna Jujeña

$\begin{array}{lll}X & X\end{array}$

$\mathrm{X}$
Morrone (2001a)

Martínez-Carretero

(1995)

Martínez-Carretero (1995) 


\section{Comentario final}

Ciertas características de los datos empleados (i.e., diversidad taxonómica y ecológica de los organismos estudiados, jerarquía sistemática de los taxa tratados (Szumik et al. 2007)), así como su estructura espacial, afectan de manera evidente el reconocimiento de patrones biogeográficos. A pesar de su relevancia, en la mayoría de los estudios biogeográficos publicados estas características no son discutidas ni informadas. Considerar las particularidades de cada conjunto de datos es importante, tanto para trazar estrategias de análisis optimas, como para efectuar una correcta interpretación de los patrones encontrados.

Por otro lado, la exploración de distintos valores de parámetros durante la búsqueda de patrones, puede brindar una idea sobre el universo de posibles resultados obtenibles a partir de un conjunto de datos, permitiéndonos obtener cierta medida cualitativa de la "estabilidad" de nuestros resultados finales.

Finalmente, la ocurrencia de diferentes procesos formadores de patrones a distintas escalas espaciales hace imprescindible la descripción de estos patrones bajo diferentes tamaños de grilla (Levin 1992, Morrone \& Escalante 2002, Willig et al. 2003). El uso de diferentes tamaños de celda durante los análisis permite el reconocimiento de áreas de endemismo con diferentes características (e.g., tamaño, composición de especies), evidenciando una diversidad de patrones que queda encubierta al utilizar una única escala de análisis.

$\mathrm{Si}$ bien existe gran cantidad de trabajos sobre la determinación de patrones biogeográficos en América del Sur (e.g., RoigJuñent 1994, Morrone 1996, Morrone et al. 2002), en la mayoría de ellos permanecen sin discusión los aspectos metodológicos mencionados. El desarrollo de trabajos que tomen en cuenta estos puntos enriquecería el conocimiento de la biogeografía de América del Sur, permitiendo reevaluar y elaborar una nueva discusión sobre los esquemas conocidos.

\section{AGRADECIMIENTOS}

Los autores agradecen al CONICET por su apoyo. Este trabajo forma parte de diversos proyectos: "biodiversidad de artrópodos montanos del centro oeste de Argentina" (PICT 01-11120), Proyecto Yamana (Fundación BBVA), "biodiversidad de artrópodos argentinos con énfasis en los estudios regionales del NOA" (CONICET-PIP 6502) y "caracterización artropodológica de las Yungas argentinas" (PICT 2002-01-12605). Agradecemos al INSUE. A Mercedes Lizarralde de Grosso y Luis Grosso por facilitar nuestro trabajo. MDC agradece al Instituto de Herpetología de la Fundación Miguel Lillo, en donde se desarrolló parte de este trabajo; a Florencia Vera Candioti, Marcos Mirande, Juan M. Morales y Salvador Arias, quienes leyeron atentamente primeras versiones de este manuscrito, y realizaron valiosos comentarios. Gabriela Quintana y Florencia Vera Candioti ayudaron en la elaboración de las figuras.

\section{LITERATURA CITADA}

ANDERSON S \& LF MARCUS (1993) Effects of quadrate size on measurements of species density. Journal of Biogeography 20: 421-428.

ARTIGAS JN (1975) Introducción al estudio por computación de las áreas zoogeográficas de Chile continental basado en la distribución de 903 especies de animales terrestres. Gayana (Chile) 4: 3-25.

AUSTIN MP (2002) Spatial prediction of species distribution: an interface between ecological theory and statistical modeling. Ecological Modelling 157: 101-118

BEAUMONT LJ, L HUGHES \& M POULSEN (2005) Predicting species distributions: use of climatic parameters in BIOCLIM and its impact on predictions of species' current and future distributions. Ecological Modelling 186: 250-269.

BRUNDIN L (1966) Transantarctic relationships and their significance, as evidence by chironomid midges, with a monograph of the subfamilies Podonominae and Aphroteniinae and the austral Heptagyiae. Kungliga Svenska Vetenskapsakadenien Handlinger 11: 1-472.

CABRERA AL \& A WILLINK (1973) Biogeografía de América Latina. Monografía 13, Serie de Biología, Organización de Estados Americanos, Washington, Distrito de Columbia, USA. 120 pp.

CRISCI JV, MM CIGLIANO, JJ MORRONE \& S ROIGJUNENT (1991) Historical biogeography of southern South America: a cladistic approach. Systematic Zoology 40: 152-171.

DARLINGTON, PJ (1965) Biogeography of the southern end of the world. Distribution and history of the far southern life and land with assessment of continental drift. Harvard University Press, Cambridge, Massachusetts, USA. 236 pp.

DOMÍNGUEZ MC, ROIG-JUÑENT S, TASSIN JJ, OCAMPO FC \& FLORES GE (2006) Areas of endemism of the Patagonian steppe: an approach based on insect distributional patterns using 
endemicity analysis. Journal of Biogeography 33: $1527-1537$.

ESCALANTE T (2005) Las bases de datos curatoriales y el estudio espacial de la biodiversidad: Un ejemplo con los mamíferos terrestres de México. En: Llorente J \& JJ Morrone (eds) Regionalización biogeográfica en Iberoamérica y tópicos afines: primeras jornadas biogeográficas de la Red Iberoamericana de Biogeografía y Entomología Sistemática: 339-350. CYTED-UNAM-Conabio, México.

GOLOBOFF P (2004) NDM/VNDM, Programs for identification of areas of endemism. Program and documentation. URL:http:www.zmuk.dk/public/ phylogeny/endemism.

HOOKER, JD (1853) Florae Novae-Zelandie. London, United Kingdom

HORTAL J, JM LOBO \& A JIMÉNEZ-VALVERDE (2007) Limitations of biodiversity databases: case study on seed-plant diversity in Tenerife, Canary Islands. Conservation Biology 21: 853-863.

HUMPHRIES, CJ (1981) Biogeographical methods and the southern beeches (Fagaceae: Nothofagus). En: Funk VA \& DR Brooks (eds) Advances in cladistics: 177-207. The New York Botanical Garden, New York, New York, USA

JEANNEL, R (1942) La Genèse des faunes terrestres. Eléments de Biogeográphie. Press Universitaires, París, Francia. 513 pp

JEANNEL R (1967) Biogeográphie de l'Amérique Austral En: Delamare Deboutteville C \& E Rapoport (eds) Biologie de 1'Amérique Australe, 2: 401-460. CNRS et CNICT, París, Francia.

KUSCHEL G (1964) Problems concerning an austral region. En: Gressit, JL, CH Lindroth, FR Forsberg, CA Fleming \& EG Turbott (eds) Pacific basin biogeography: 443-449. Bishop Museum Press, Honolulu, Hawaii, USA.

KUSCHEL G (1969) Biogeography and ecology of South American Coleoptera. En: Fittkau EJ, J Illies, H Klinge, GH Schwabe \& H Sioli (eds) Biogeography and ecology in South America, 2: 709-722. Dr. Junk Publishers, The Hague, The Netherlands.

LAFFAN SW \& CRISP MD (2003) Assessing endemism at multiple spatial scales, with an example from the Australian vascular flora. Journal of Biogeography 30: 511-520.

LEVIN S (1992) The problem of pattern and scale in ecology. Ecology 73: 1943-1967.

MARTÍNEZ-CARRETERO E (1995) La Puna argentina: delimitación general y división en distritos florísticos. Boletín de la Sociedad Argentina de Botánica (Argentina) 31: 27-40.

MONRÓS F (1958) Consideraciones sobre la fauna del sur de Chile y revisión de la tribus Stenomelini (Coleoptera, Chrysomelidae). Acta Zoológica Lilloana (Argentina) 15: 143-153.

MORRONE JJ (1996) The biogeographic Andean subregion: a proposal exemplied by arthropod taxa (Arachnida, Crustacea, and Hexapoda). Neotropica 42: 103-114.

MORRONE JJ (2001a) Biogeografía de América Latina y el Caribe. Manuales \& Tesis. Sociedad Entomológica Aragonesa, Zaragoza, España.

MORRONE JJ (2001b) Review of the biogeographic provinces of the Patagonian subregion. Revista de la Sociedad Entomológica Argentina 60: 1-8.

MORRONE JJ \& T ESCALANTE (2002) Parsimony analysis of endemicity (PAE) of Mexican terrestrial mammals at different area units: when size matters. Journal of Biogeography 29: 1-10.
MORRONE JJ, S ROIG-JUNENT \& GE FLORES (2002) Delimitation of biogeographic districts in central Patagonia (southern South America), based on beetle distributional patterns (Insecta: Coleoptera: Carabidae and Tenebrionidae). Revista del Museo Argentino de Ciencias Naturales (Argentina) 4: 1-6.

MORRONE JJ (2006) Biogeographic areas and transition zones of Latin America and the Caribbean Islands based on panbiogeographic and cladistic analyses of the entomofauna. Annual Review of Entomology 51: 467-494.

MYERS N, RA MITTERMEIER, G MITTERMEIER, DA FONSECA GAB \& J KENT (2000) Biodiversity hotspots for conservation priorities. Nature 403: 853-858.

PHILLIPS SJ, RP ANDERSON \& RE SCHAPIRE (2006) Maximum entropy modeling of species geographic distributions. Ecological Modelling 190: 231-259.

PIZARRO-ARAYA J \& V JEREZ (2004) Distribución geográfica del género Gyriosomus Gérin-Méneville, 1834 (Coleoptera: Tenebrionidae): una aproximación biogeográfica. Revista Chilena de Historia Natural 77: 491-500.

POSADAS P \& D MIRANDA-ESQUIVEL (1999) El PAE (Parsimony analysis of endemicity) como una herramienta en la evaluación de la biodiversidad. Revista Chilena de Historia Natural 72: 539-546.

RINGUELET R (1961) Rasgos fundamentales de la zoogeografía de la Argentina. Physis (Argentina) 22: 151-188

ROIG-JUÑENT S (1994) Historia biogeográfica de América del Sur austral. Multequina 3: 167-203.

ROIG-JUÑENT S \& GE FLORES (2001) Historia biogeográfica de las áreas áridas de América del Sur austral. En: Llorente Bousquets J \& JJ Morrone (eds) Introducción a la biogeografía en Latinoamérica: teorías, conceptos, métodos y aplicaciones: 257-266. Las Prensas de Ciencias, Facultad de Ciencias, UNAM, México.

ROIG-JUÑENT S, JV CRISCI, P POSADAS \& S LAGOS (2002) Áreas de distribución y de endemismo en zonas continentales. En: Costa C, SA Vanin, JM Lobo \& A Melic (eds) Proyecto de red iberoaméricana de biogeografía y entomología sistemática, PrIBES, 2: 247-266. Monografías del Tercer Milenio, Sociedad. Entomológica Aragonesa (SEA) y CYTED, Zaragoza, España.

ROIG-JUÑNET S, GE FLORES \& C MATTONI (2003) Consideraciones biogeográficas de la precordillera (Argentina) basadas en artrópodos epígeos. En: Morrone JJ \& J Llorente Bousquets (eds) Una perspectiva latinoamericana de la biogeografía: 275-288. Las Prensas de Ciencias, Facultad de Ciencias, UNAM, México DF, México.

ROIG-JUÑENT S, MC DOMÍNGUEZ, GE FLORES \& C MATTONI (2005) Biogeographic history of South american arid lands: a view from its arthropods. Journal of Arid Environments 66: 404-420.

ROIG-JUÑENT S, MF TOGNELLI \& JJ MORRONE (2008) Aspectos biogeográficos de la entomofauna argentina. En: Claps LE, G Debandi \& S RoigJuñent (en prensa) Diversidad de artrópodos argentinos, 2: 11-30. Sociedad Entomológica, Tucumán, Argentina.

SKOSTTBERG C (1960) Remarks on the plant geography of the southern temperate zone. Proceedings of the Royal Society of London B 152: 447-457.

SZUMIK C \& P GOLOBOFF (2004) Areas of endemism: an improved optimality criterion. Systematic Biology 53: 968-977. 
SZUMIK CA, F CUEZZO, P GOLOBOFF \& AE CHALUP (2002) An optimality criterion to determine areas of endemism. Systematic Biology 51: 806-816.

SZUMIK C \& S ROIG-JUNENT (2005) Criterio de optimación para áreas de endemismo: el caso de América del Sur austral. En: Llorente-Bousquets J \& JJ Morrone (eds) Regionalización biogeográfica en Iberoamérica y tópicos afines, 2: 495-508. Las Prensas de Ciencias, Facultad de Ciencias, UNAM, Distrito Federal, México.

SZUMIK C, D CASAGRANDA \& ROIG-JUÑENT (2006) Manual de NDM/VNDM: programas para la identificación de áreas de endemismo. URL:http:// www.zmuc.dk/public/phylogeny/endemism/

SZUMIK C, S NOMDEDEU, A PANIZZA, L AAGESEN,
D CASAGRANDA, F NAVARRO, JM DÍAZGÓMEZ, M SANDOVAL, D BALDO, G SAN BLAS, F CUEZZO, L TAHER, P GOLOBOFF, M LIZARRALDE \& F ZULOAGA (2007) Biogeografía del norte argentino (paralelos 21 a 32): primer ensayo utilizando vertebrados, insectos y plantas. Darwiniana (Suplemento) 45: 49-51.

WILLIG MR, DM KAUFMAN \& RD STEVENS (2003) Latitudinal gradients of biodiversity: pattern, process, scale, and synthesis. Annual Review of Ecology and Systematics 34: 273-309.

ZANIEWSKI AE, A LEHMANN \& JMCC OVERTON (2002) Predicting species spatial distributions using presence-only data: a case study of native New Zealand ferns. Ecological Modelling 157: 261-280.

Editor Asociado: Patricio Camus

Recibido el 30 de mayo de 2008; aceptado el 10 de septiembre de 2008 


\section{APÉNDICE 1}

Lista de números de identificación asignado a cada especie

List of identification number assigned to each species

\begin{tabular}{|c|c|c|c|}
\hline Número & Nombre de la especie & Número & Nombre de la especie \\
\hline 0 & Barypus bonvouloiri & 44 & Ceroglossus chilensis villaricensis \\
\hline 1 & Barypus parallelus & 45 & Ceroglossus chilensis selanodicus \\
\hline 2 & Barypus aequicostis & 46 & Ceroglossus chilensis latemarginatus \\
\hline 3 & Barypus calchaquensis & 47 & Ceroglossus chilensis mochae \\
\hline 4 & Barypus comechingonensis & 48 & Ceroglossus chilensis solieri \\
\hline 5 & Barypus pulchellus & 49 & Ceroglossus intermedius \\
\hline 6 & Barypus rivalis & 50 & Ceroglossus darwini darwini \\
\hline 7 & Barypus speciosus & 51 & Ceroglossus darwini morawitzi \\
\hline 8 & Barypus chubutensis & 52 & Ceroglossus darwini gerstaeckeri \\
\hline 9 & Barypus clivinoides & 53 & Ceroglossus darwini magellanicus \\
\hline 10 & Barypus dentipenis & 54 & Ceroglossus sp.eciosus \\
\hline 11 & Barypus deplanatus & 55 & Ceroglossus suturalis suturalis \\
\hline 12 & Barypus gentilii & 56 & Ceroglossus suturalis reichei \\
\hline 13 & Barypus giaii & 57 & Ceroglossus sybarita \\
\hline 14 & Barypus longitarsis & 58 & Ceroglossus valdiviae valdiviae \\
\hline 15 & Barypus mendozensis & 59 & Ceroglossus valdiviae lepidus \\
\hline 16 & Barypus minus & 60 & Ceroglossus valdiviae inexpectatus \\
\hline 17 & Barypus neuquensis & 61 & Ceroglossus valdiviae chiloénsis \\
\hline 18 & Barypus painensis & 62 & Ceroglossus valdiviae subnitens \\
\hline 19 & Barypus schajovskoii & 63 & Ceroglossus valdiviae andestus \\
\hline 20 & Microbarypus silvicola & 64 & Ceroglossus valdiviae elegantissimus \\
\hline 21 & Nothobroscus chilensis & 65 & Cnemalobus araucanus \\
\hline 22 & Creobius eydouxii & 66 & Cnemalobus bruchi \\
\hline 23 & Creobius gravessi & 67 & Cnemalobus convexus \\
\hline 24 & Cascellius septentrionalis & 68 & Cnemalobus coquimbanus \\
\hline 25 & Nothocascellius aeneoniger & 69 & Cnemalobus curtisii \\
\hline 26 & Nothocascellius hyadessi & 70 & Cnemalobus cyaneus \\
\hline 27 & Monolobus testaceus & 71 & Cnemalobus cylindricus \\
\hline 28 & Monolobus ovalipennis & 72 & Cnemalobus deplanatus \\
\hline 29 & Migadops latus & 73 & Cnemalobus desmarestii \\
\hline 30 & Pseudomigadops ater & 74 & Cnemalobus gentilii \\
\hline 31 & Pseudomigadops darwini & 75 & Cnemalobus germaini \\
\hline 32 & Pseudomigadops falklandicus & 76 & Cnemalobus hirsutus \\
\hline 33 & Pseudomigadops nigrocoeruleus & 77 & Cnemalobus litorales \\
\hline 34 & Pseudomigadops ovalis & 78 & Cnemalobus mapuche \\
\hline 35 & Rhytidognathus ovalis & 79 & Cnemalobus mendozensis \\
\hline 36 & Antarctonomus complanatus & 80 & Cnemalobus montanus \\
\hline 37 & Migadopidius bimaculatus & 81 & Cnemalobus neuquensis \\
\hline 38 & Lissopterus hyadessi & 82 & Cnemalobus nuria \\
\hline 39 & Lissopterus quadrinotatus & 83 & Cnemalobus obscurus \\
\hline 40 & Ceroglossus chilensis chilensis & 84 & Cnemalobus pegnai \\
\hline 41 & Ceroglossus chilensis gloriosus & 85 & Cnemalobus piceus \\
\hline 42 & Ceroglossus chilensis jaffueli & 86 & Cnemalobus pulchellus \\
\hline 43 & Ceroglossus chilensis teтисеnsis & 87 & Cnemalobus reichardti \\
\hline 88 & Cnemalobus striatipennis & 135 & Notaphiellus obliteratus \\
\hline 89 & Cnemalobus striatus & 136 & Notaphiellus solieri \\
\hline 90 & Cnemalobus substriatus & 137 & Notholopha punctigerum \\
\hline 91 & Cnemalobus sulciferus & 138 & Notholopha sexfoveolatum \\
\hline 92 & Cnemalobus tupungatensis & 139 & Notholopha kuscheli \\
\hline 93 & Nothanillus germaini & 140 & Notholopha melanopodum \\
\hline 94 & Nothanillus luisae & 141 & Notholopha scitulum \\
\hline 95 & Pericompsus circuliformis & 142 & Notholopha stricticolle \\
\hline 96 & Paranillopsis piguensis & 143 & Pseudotrepanes derbesi \\
\hline 97 & Paranillopsis pampensis & 144 & Nothophena lonae \\
\hline 98 & Bembicidium cordillerae & 145 & Nothophena pollidiguttula \\
\hline 99 & Bembicidium duvali & 146 & Peryphus eburneoniger \\
\hline
\end{tabular}


Apéndice 1 (Continuación)

\begin{tabular}{|c|c|c|c|}
\hline Número & Nombre de la especie & Número & Nombre de la especie \\
\hline 100 & Bembicidium strobeli & 147 & Peryphus engelharti \\
\hline 101 & Bembidium tesselatum & 148 & Peryphus philippi \\
\hline 102 & Notaphus convergens & 149 & Peryphus ringueleti \\
\hline 103 & Notaphus deplanatus & 150 & Peryphus rufoplagiatus \\
\hline 104 & Notaphus kuscheli & 151 & Peryphus tucumanus \\
\hline 105 & Notaphus luridus & 152 & Peryphus chilensis \\
\hline 106 & Notaphus aricensis & 153 & Peryphus kaszabi \\
\hline 107 & Notaphus bicolor & 154 & Peryphus maculifer \\
\hline 108 & Notaphus gabrielus & 155 & Peryphus nivalis \\
\hline 109 & Notaphus unifasciatus & 156 & Peryphus sp.inolae \\
\hline 110 & Notaphus aubei & 157 & Peryphus $\mathrm{n}$ sp. 2 \\
\hline 111 & Notaphus bonariense & 158 & Peryphus mendocinus \\
\hline 112 & Notaphus calverti & 159 & Peryphus orregoy \\
\hline 113 & Notaphus chaudoirianus & 160 & Peryphus $\mathrm{n}$ sp. 1 \\
\hline 114 & Notaphus fischeri & 161 & Plocamoperyphus mandibularis \\
\hline 115 & Notaphus inconstans & 162 & Notoperyphus angelieri angelieri \\
\hline 116 & Notaphus jacobseni & 163 & Notoperyphus cekalovici \\
\hline 117 & Notaphus laticolle & 164 & Bembidarenas setiventre \\
\hline 118 & Notaphus mirasoi & 165 & Pycnochila fallaciosa \\
\hline 119 & Notaphus olivieri & 166 & Plagiotelum irinum \\
\hline 120 & Notaphus posticalis & 167 & Anisostichus amoenus \\
\hline 121 & Notaphus stenoderus & 168 & Anisostichus laevis laevis \\
\hline 122 & Nothocys coerulescens & 169 & Anisostichus laevis bolivianus \\
\hline 123 & Nothocys delamarei & 170 & Anisostichus octopunctatus \\
\hline 124 & Nothocys grossepunctatus & 171 & Anisostichus posticus \\
\hline 125 & Nothocys marcidus & 172 & Criniventer rufus \\
\hline 126 & Nothocys marginatus & 173 & Allendia chilensis \\
\hline 127 & Nothocys nigrita & 174 & Nemaglossa brevis \\
\hline 128 & Nothocys nitidus & 175 & Bradycellus arcobasis \\
\hline 129 & Nothocys topali & 176 & Bradycellus chilensis \\
\hline 130 & Nothocys silvicola & 177 & Bradycellus impressifrons \\
\hline 131 & Notaphiellus aereus & 178 & Bradycellus foveicollis \\
\hline 132 & Notaphiellus cekalovici & 179 & Bradycellus tibialis \\
\hline 133 & Notaphiellus cupreostriatus & 180 & Bradycellus ruficollis \\
\hline 134 & Notaphiellus hornensis & 181 & Bradycellus unistriatus \\
\hline 182 & Paramecus cylindricus & 226 & Mimophilorhizus chilensis \\
\hline 183 & Paramecus laevigatus & 227 & Catascopellus crassipes \\
\hline 184 & Trachysarus antarcticus & 228 & Lebia azurea \\
\hline 185 & Trachysarus basalis & 229 & Dromius elongatulus \\
\hline 186 & Trachysaurus bicolor & 230 & Dromius giachinoi \\
\hline 187 & Trachysarus emdeni & 231 & Dromius sulcatulus \\
\hline 188 & Trachysarus kuscheli & 232 & Dromius maculipennis \\
\hline 189 & Trachysarus ovalipennis & 233 & Axinopalpus cruseoi \\
\hline 190 & Trachysarus neopallipes & 234 & Axinopalpus delicatulus \\
\hline 191 & Trachysarus punctiger & 235 & Axinopalpus humeralis \\
\hline 192 & Trachysarus sericeus & 236 & Eutogenius fuscus \\
\hline 193 & Polpochila monrosi & 237 & Austroglyptolenus mendozensis \\
\hline 194 & Polpochila chilensis & 238 & Austroglyptolenus precordillerae \\
\hline 195 & Euproctinus fasciatus & 239 & Incagonum ambiguum \\
\hline 196 & Mimodromius (Mimodromius) chilensis & 240 & Incagonum chilense \\
\hline 197 & Mimodromius chopardi & 241 & Incagonum coquimbanum \\
\hline 198 & Mimodromius cyanipennis & 242 & Incagonum cordicolle \\
\hline 199 & Mimodromius elegantulus & 243 & Incagonum discosulcatum \\
\hline 200 & Mimodromius fuscus & 244 & Incagonum fuscoaeneum \\
\hline 201 & Mimodromius lividus & 245 & Incagonum gayi \\
\hline 202 & Mimodromius metallicus & 246 & Incagonum lineatopunctatum \\
\hline 203 & Mimodromius obscuripennis & 247 & Incagonum melas \\
\hline 204 & Mimodromius philippii & 248 & Incagonum quadricolle \\
\hline 205 & Mimodromius bicolor & 249 & Incagonum semistriatum \\
\hline 206 & Mimodromius gracilis & 250 & Tropopterus giraudi \\
\hline
\end{tabular}


Apéndice 1 (Continuación)

\begin{tabular}{|c|c|c|c|}
\hline Número & Nombre de la especie & Número & Nombre de la especie \\
\hline 207 & Mimodromius in sp.eratus & 251 & Bembidiomorphum convexum \\
\hline 208 & Mimodromius nigroeburneus & 252 & Ogmopleura blandus \\
\hline 209 & Mimodromius phaeoxanthus & 253 & Ogmopleura meticulosus \\
\hline 210 & Mimodromius bolivianus & 254 & Ogmopleura obscuripennis \\
\hline 211 & Mimodromius parallelus & 255 & Ogmopleura sommians \\
\hline 212 & Mimodromius cruciger & 256 & Feroniola bradytoides \\
\hline 213 & Mimodromius flessi & 257 & Feroniola famelica \\
\hline 214 & Mimodromius martinezi & 258 & Feroniola laticollis \\
\hline 215 & Mimodromites nigrotestaceus & 259 & Feroniola minor \\
\hline 216 & Mimodromites (Mimodromites) guttula & 260 & Feroniola montevidiana \\
\hline 217 & Mimodromites (Mimodromites) cyaneus & 261 & Feroniola puncticollis \\
\hline 218 & Apterodromites saizi & 262 & Feroniola subsinuata \\
\hline 219 & Cyanotarsus andinus & 263 & Parhypates abacetoides \\
\hline 220 & Cyanotarsus foveolatus & 264 & Parhypates bonariensis \\
\hline 221 & Falsodromius erythropus & 265 & Parhypates chilensis chilensis \\
\hline 222 & Chaudoirina nigrofasciata & 266 & Parhypates chilensis chubutensis \\
\hline 223 & Chaudoirina orfilai & 267 & Parhypates chilensis ardens \\
\hline 224 & Chaudoirina vianai & 268 & Parhypates depressulus \\
\hline 225 & Carboniella platensis & 269 & Parhypates oblitus \\
\hline 270 & Parhypates uruguaycus uruguaycus & 317 & Metius chilensis \\
\hline 271 & Parhypates uruguaycus semisericeus & 318 & Metius circumfusus \\
\hline 272 & Parhypates extenuatus & 319 & Metius cordatus \\
\hline 273 & Parhypates bonelli bonelli & 320 & Metius brunnescens \\
\hline 274 & Parhypates bonelli chiloensis & 321 & Metius eurypterus \\
\hline 275 & Parhypates chalybicolor & 322 & Metius femoratus \\
\hline 276 & Parhypates nunni & 323 & Metius flavipes \\
\hline 277 & Parhypates rufipalpis & 324 & Metius flavipleuris \\
\hline 278 & Parhypates stenomus & 325 & Metius gigas \\
\hline 279 & Parhypates sinuatipennis & 326 & Metius gilvipes \\
\hline 280 & Parhypates convexipenne & 327 & Metius harpaloides \\
\hline 281 & Parhypates cordicollis & 328 & Metius kuscheli \\
\hline 282 & Trirammatus aerea & 329 & Metius kulti \\
\hline 283 & Trirammatus chalceus & 330 & Metius latigastrichus \\
\hline 284 & Trirammatus glauca & 331 & Metius malachiticus malachiticus \\
\hline 285 & Trirammatus lucidus & 332 & Metius malachiticus septentrionalis \\
\hline 286 & Trirammatus lucidus argentina & 333 & Metius marginatus \\
\hline 287 & Trirammatus moerens & 334 & Metius motschulskyi \\
\hline 288 & Trirammatus nebrioides & 335 & Metius obscurus \\
\hline 289 & Trirammatus striatula & 336 & Metius ovalipennis \\
\hline 290 & Trirammatus subcorynthia & 337 & Metius parvicollis \\
\hline 291 & Trirammatus violaceus & 388 & Metius pogonoides \\
\hline 292 & Trirammatus niki & 339 & Metius puncticollis \\
\hline 293 & Trirammatus rufescens & 340 & Metius robustus \\
\hline 294 & Trirammatus currens & 341 & Metius rotundatus \\
\hline 295 & Trirammatus chaudoiri & 342 & Metius rotundicollis \\
\hline 296 & Trirammatus guerini & 343 & Metius silvetri \\
\hline 297 & Trirammatus unistriatus unistriatus & 344 & Metius subcoeruleus \\
\hline 298 & Trirammatus unistriatus noticolor & 345 & Systolosoma breve \\
\hline 299 & Trirammatus loxandroides & 346 & Systolosoma lateriticum \\
\hline 300 & Trirammatus pseudoharpalus & 347 & Thalassobius testaceus \\
\hline 301 & Trirammatus vagans & 348 & Kenodactyllus audouini \\
\hline 302 & Trirammatus selkirki & 349 & Trechisibus antarcticus antarcticus \\
\hline 303 & Trirammatus lacordairei & 350 & Trechisibus antarcticus racovitzai \\
\hline 304 & Pterostichus skottsbergi & 351 & Trechisibus antarcticus longipennis \\
\hline 305 & Feronia ignobilis & 352 & Trechisibus backstroemi \\
\hline 306 & Pterostichus kuscheli & 353 & Trechisibus bruchi \\
\hline 307 & Abropus carnifex & 354 & Trechisibus cekalovici \\
\hline 308 & Metius aeneus & 355 & Trechisibus chloroticus \\
\hline 309 & Metius amaroides & 356 & Trechisibus collaris \\
\hline 310 & Metius andicola & 357 & Trechisibus cristinensis \\
\hline
\end{tabular}


Apéndice 1 (Continuación)

\begin{tabular}{|c|c|c|c|}
\hline Número & Nombre de la especie & Número & Nombre de la especie \\
\hline 311 & Metius annulicornis & 358 & Trechisibus darwini \\
\hline 312 & Metius blandus & 359 & Trechisibus depressus \\
\hline 313 & Metius bonaerensis & 360 & Trechisibus falklandicus \\
\hline 314 & Metius canotae & 361 & Trechisibus femoralis femoralis \\
\hline 315 & Metius carnifex & 362 & Trechisibus femoralis depressicollis \\
\hline 316 & Metius caudatus & 363 & Trechisibus geae \\
\hline 364 & Trechisibus kuscheli & 395 & Aemalodera dentimaculata kuscheli \\
\hline 365 & Trechisibus kamergei & 396 & Aemalodera limbata limbata \\
\hline 366 & Trechisibus monrosi & 397 & Aemalodera limbata bruneipennis \\
\hline 367 & Trechisibus magellanus & 398 & Aemalodera limbata trasandina \\
\hline 368 & Trechisibus nigripennis nigripennis & 399 & Aemalodera testacea testacea \\
\hline 369 & Trechisibus nigripennis scapularis & 400 & Aemalodera testacea depressa \\
\hline 370 & Trechisibus nigripennis chilotus & 401 & Homaloderes germaini \\
\hline 371 & Trechisibus nigripennis australis & 402 & Homaloderes jacquesi \\
\hline 372 & Trechisibus nitidus & 403 & Pseudocnides monolcus \\
\hline 373 & Trechisibus obtusiusculus & 404 & Pseudocnides rugosifrons \\
\hline 374 & Trechisibus oreobates & 405 & Pseudocnides solieri \\
\hline 375 & Trechisibus politus politus & 406 & Pseudocnides patagonica \\
\hline 376 & Trechisibus politus delfini & 407 & Oxytrechus arechavaletai \\
\hline 377 & Trechisibus rectangulus & 408 & Trechinotus flavocinctus \\
\hline 378 & Trechisibus setulosus & 409 & Trechinotus flovolimbatus \\
\hline 379 & Trechisibus sinuatus & 410 & Trechinotus striatulus \\
\hline 380 & Trechisibus stricticollis & 411 & Merizodus angusticollis \\
\hline 381 & Trechisibus topali & 412 & Merizodus catapilenaus \\
\hline 382 & Trechisibus alexius & 413 & Merizodus soledadinus \\
\hline 383 & Trechisibus ciffaiti & 414 & Chaltenia patagonica \\
\hline 384 & Trechisibus straneoi & 415 & Tropopsis marginicollis \\
\hline 385 & Trechisibus saizi & 416 & Tropopsis biguttata \\
\hline 386 & Trechisibus brachyderus & 417 & Tropopsis punctatostriatus \\
\hline 387 & Trechisibus cyclopterus & 418 & Tetragonoderus viridis \\
\hline 388 & Trechisibus nahuelanus & 419 & Tetragonoderus viridis cekalovici \\
\hline 389 & Trechisibus tucumanus & 420 & Cicindela chilensis \\
\hline 390 & Nothotrechisibus hornensis hornensis & 421 & Cicindela gomarzi \\
\hline 391 & Nothotrechisibus hornensis brunswickensis & 422 & Cicindela vidali \\
\hline 392 & Trechichomimus aphaenops & 423 & Mimodromius straneoi \\
\hline 393 & Aemalodera centromaculata & 424 & Mimodromius trivittis \\
\hline 394 & Aemalodera dentimaculata dentimaculata & 425 & Bembidarenas reicheellum \\
\hline
\end{tabular}

